\title{
Smart Investment for Redundancies Selection Integrated to Reconfigurable Fault-Tolerant Control Design
}

\author{
P. A. Luppi* ${ }^{* \dagger, \ddagger}$ and M. S. Basualdo*,\$, II \\ ${ }^{\dagger}$ CIFASIS, CONICET - UNR, 27 de Febrero 210 bis, S2000EZP Rosario, Argentina \\ ${ }^{\ddagger}$ FCEIA, Universidad Nacional de Rosario, Pellegrini 250, S2000EZP Rosario, Argentina \\ IIUniversidad Tecnológica Nacional - FRRo, Zeballos 1341, S2000BQA Rosario, Argentina \\ Supporting Information
}

ABSTRACT: This paper presents a methodology for the optimal hardware redundancies selection in the context of reconfigurable fault-tolerant control design. Assuming that a nominal controller of reduced dimension has been successfully designed, the objective is to accommodate a presumed set of failures, including partial, total, and simultaneous actuator faults, preserving the system stability and maintaining an acceptable dynamic performance. The methodology is based on a multiobjective optimization framework to obtain a suitable trade-off between conflicting design objectives such as controllability and performance. The selection of additional hardware devices (not included in the nominal controller) is penalized to determine the minimum number of redundancies that should be installed. In addition, the redundancies selection is performed without explicitly considering the type of control structure. The effectiveness of the proposed approach is tested using the well-known Tennessee Eastman benchmark.

\section{INTRODUCTION}

In any automated industrial process, the occurrence of failures can cause the control system malfunction. This could endanger the personnel safety, damage physical equipment, and increase the idle time of the process, which implies significant economic losses. ${ }^{1}$ This generates a strong motivation for the development of fault-tolerant control (FTC) systems. Their main objective is to preserve the system stability and maintain an acceptable dynamic performance in order to avoid a process shutdown, conferring high availability. ${ }^{2,3}$ In general, there are two different ways for handling failures. One approach is based on a rigid controller structure, designed to be robust for a specific set of faults. The other methodology performs a real-time reconfiguration of the nominal controller to accommodate the faults by implementing the appropriate control actions. The first approach is called passive FTC, while the second one corresponds to active (or reconfigurable) FTC. ${ }^{4}$ Beyond their significant differences, both strategies heavily depend on system redundancies to achieve fault tolerance. ${ }^{2,5}$ The design of FTC systems requires solving several issues, including ${ }^{2,6}$ (i) the overall system architecture, (ii) the trade-off between conflicting design objectives (stability, performance, hardware cost, etc.), (iii) the management of system redundancies, and (iv) the development and implementation of the controller to achieve the design objectives, subject to an efficient utilization of the redundancies.

As it is well-known, in multivariable processes, the interaction among different variables can cause the reduction of the system stability margin (or even instability) when certain failures occur. In this context and considering reconfigurable fault-tolerant structures, Luppi et al. ${ }^{7}$ proposed a procedure for the design of the nominal controller (NC) characterized by (i) reduced dimension, ensuring availability of additional degrees of freedom, (ii) being fully decentralized, contributing to its structural flexibility and fault-tolerant capability, ${ }^{8}$ and (iii) fulfillment of a sufficient condition for decentralized integral controllability (DIC), ${ }^{9}$ which states that a decentralized structure with integral action in each loop remains stable despite some loops being detuned or taken out of service due to failures. The aim of the present paper is to develop a complementary procedure for the methodology detailed in Luppi et al., 7 giving a complete strategy for synthesizing reconfigurable FTC structures. When specific abnormal events occur, the resulting dynamic performance could be unacceptable although the NC design can guarantee the stability of the system. For this case, the reconfiguration of the NC structure should be considered. In this work, the design of the reconfigurable control structure is addressed together with the low cost hardware redundancies selection in order to accommodate a preconceived set of actuator failures.

A variety of papers concerning the design of FTC systems have been published, in which the available degrees of freedom are exploited with control purposes. However, few studies focus on a rigorous analysis of the system redundancy. ${ }^{2,4}$ In this regard, it remains an interesting challenge to propose criteria and

Received: April 20, 2016

Revised: August 9, 2016

Accepted: August 13, 2016

Published: August 14, 2016 
procedures that allow the (optimal) redundancies selection of actuators and sensors. Jiang and Zhao ${ }^{5}$ presented a reliable control system design methodology for managing actuator failures, where the redundancies were explicitly defined in the framework of multiple-input/multiple-output (MIMO) systems. However, the proposed design only considered the case of multiple-input/single-output (MISO) systems. It must be noted that generally the selection of an excessive number of actuators and sensors does not ensure a good performance of the control system. ${ }^{10,11}$ Moreover, this can impact negatively on the investment and operating costs. Another issue is that many optimization-based contributions try to achieve the optimal value of a single functional cost. ${ }^{12}$ This contrasts with the fact that the overall performance of a designed system is typically analyzed by taking into account multiple indexes. ${ }^{7,13}$ On the other hand, only structural faults such as actuator blockade and loss of sensor measurements were analyzed in a previous work. ${ }^{12}$ The proposed framework did not allow the treatment of partial failures, such as the reduction of actuator effectiveness. ${ }^{4,14}$ In this context, the present paper proposes a methodology for obtaining a minimal set of redundancies as well as the reconfigurable controller design for MIMO processes. When it is possible, the designed structures are able to accommodate partial, total, and simultaneous actuator failures. This is done based on a multiobjective optimization framework implemented with genetic algorithm, ${ }^{15}$ which allows one to obtain a suitable trade-off between controllability, performance, and investment cost.

Initially, the availability of a NC structure developed for the fault-free operation mode, which has reduced dimension, is assumed. For this purpose, the procedure presented in Luppi et al. ${ }^{7}$ can be considered. The goal of a reduced design is to ensure the existence of unused sensors and actuators. Thus, the problem of redundancies selection is feasible. In addition to the wellknown functional controllability criterion, ${ }^{16}$ this work incorporates an alternative index for measuring controllability. Morari ${ }^{17}$ introduced the resiliency concept, which was then considered by Luyben ${ }^{18}$ as a degree of controllability. Morari ${ }^{17}$ stated that a process is more resilient when the minimum singular value (MSV) of its transfer function matrix is larger. This index represents an useful tool for comparing alternative manipulated (MV) and controlled variables (CV) selections and is utilized here in order to establish the best controllable options. Note that the MSV does not depend on the pairing between the CVs and MVs. On the other hand, the optimal redundancies selection is also based on the quantification of the sum of the steady-state squared deviations (SSD) of the uncontrolled variables when certain set points and disturbances occur. ${ }^{10,19,20}$ In this paper, an extended version of this methodology is presented, which incorporates the treatment of partial actuator failures that can be modeled through an effectiveness matrix. These controllability and performance measures are utilized to formulate a set of optimization problems, resulting in a multiobjective optimization framework efficiently solved with genetic algorithm. Furthermore, the minimum number of redundancies that should be installed is analyzed. Usually this analysis is not addressed because the trend is to employ all the available control components. To this end, an objective function is proposed that penalizes the selection of additional hardware devices not included in the NC structure. It is inspired on a previous work concerning the design of fault detection and diagnosis systems. $^{21}$

The presented methodology allows one to obtain candidate hardware sets such that the selections are done without explicitly considering the type of control structure, namely, diagonal, sparse, or full. For simplicity, diagonal structures based on singleinput/single-output (SISO) proportional-integral-derivative (PID) controllers were implemented in this work. However, more complex structures can be considered in order to improve system performance. In this context, Zumoffen ${ }^{10}$ proposes the design of alternative structure types based on a previous hardware selection. On the other hand, unlike a previous work, ${ }^{7}$ here the hardware selection procedure is not integrated with the pairing task. In fact, the MSV and SSD indexes are independent from the control configuration, that is, the input-output interconnections. The pairing task was only included with the purpose of implementing the SISO PID controllers. It is considered that the procedure indicated in Kariwala and $\mathrm{Cao}^{22}$ is suitable for such purpose.

The overall approach is tested on the well-known Tennessee Eastman (TE) benchmark. ${ }^{23}$ The dynamic performance of the FTC designs is analyzed in detail by performing closed-loop simulations of the TE model subject to a set of critical actuator failures and disturbance profiles recommended by Downs and Vogel. ${ }^{23}$ Based on the temporal responses of the system, several performance indexes are calculated in order to show the potentiality of the FTC and to draw conclusions.

The paper is organized as follows. Section 2 presents the complete methodology comprising the selection of the redundancies and the design of the alternative control structures. The procedure involves several tasks such as (i) fault modeling (section 2.1), (ii) the definition of performance, controllability, and hardware cost measures (sections 2.2, 2.3, and 2.5, respectively), (iii) the formulation of the multiobjective optimization framework (section 2.4), and (iv) the input-output variables pairing in order to synthesize the control structures (section 2.6). Section 3 continues with the application of the methodology in order to design FTC systems for the TE case study. In sections 3.1.1 to 3.1.4, four fault scenarios are analyzed, comprising partial, total, and simultaneous actuator failures. Then, a complete closed-loop analysis is included in section 3.2. Finally, the conclusions are provided in section 4 .

\section{INPUT-OUTPUT REDUNDANCIES SELECTION}

When the design of a reconfigurable FTC system is considered, its performance must be analized in two different operating modes: (i) normal operation (i.e., the fault-free case), where the main objective is to achieve the best possible dynamic performance; (ii) failure scenarios, for which a stable operation and acceptable (but usually degraded) dynamic behavior is desired. Typically, for this kind of control system, the design of the following is crucial: ${ }^{2,6}$

- the nominal controller (NC), which can be easily reconfigured

- the fault detection and diagnosis (FDD) scheme, sensitive to the abnormal events

- the reconfiguration module, for the implementation of alternative control structures subject to FDD uncertainties and temporal constraints

The basic architecture of a reconfigurable FTC system is shown in Figure 1.

For the fault-free case, it is not necessary to modify the original control strategy. The nominal controller must attenuate the disturbances and ensure set point tracking and other closed-loop requirements. In Luppi et al., 7 the design of a fully decentralized NC structure was proposed. The main contribution was to ensure the system stability during the FDD phase, through the 


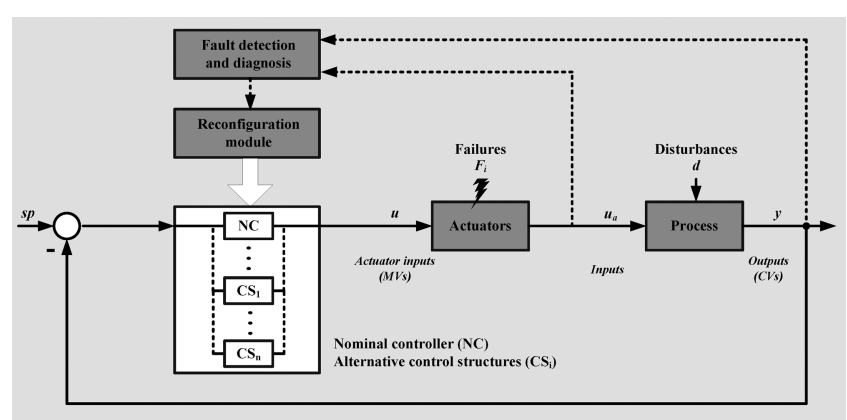

Figure 1. Reconfigurable FTC system architecture.

compliance of a sufficient condition for decentralized integral controllability. As in Luppi et al., ${ }^{7}$ a perfect FDD system is considered available; hence the design and implementation of a FDD scheme will not be discussed in this paper. A complete classification of the FDD techniques and its analysis can be found in Isermann, ${ }^{24}$ Zhang and Jiang, ${ }^{2}$ and Venkatasubramanian et al. ${ }^{25}$

On the other hand, for each considered failure scenario, two main steps involved in the reconfiguration problem must be analyzed:

1. the selection of the input-output control variables, particularly the redundancies

2. based on the selected hardware, the design of the alternative control structures

Generally, a NC reconfiguration is required when critical failures affect the system. When this is the case, usually alternative sensors and actuators must be available in order to preserve the controllability and performance. Based on these redundancies, the main objective of the reconfigurable FTC system is to synthesize alternative control structures $\left(\mathrm{CS}_{i}\right)$ in order to manage the failures. ${ }^{2,6}$ In this work, critical failures such as partial, total, and simultaneous actuator malfunctions are considered for the reconfiguration problem. The next sections present some performance and controllability criteria to define the selection of the redundancies, trying to reduce the hardware investment. The entire procedure is illustrated in Figure 2, and it is described step by step in the following sections.

2.1. Fault Modeling. Given a (stabilized) process with $n$ inputs and $m$ outputs, the system can be represented as

$$
\mathbf{y}(s)=\mathbf{G}(s) \mathbf{u}_{\mathrm{a}}(s)
$$

where $\mathbf{y}(s)$ is the output vector with dimension $m \times 1$, and $\mathbf{G}(s)$ is the process transfer function matrix with dimension $m \times n$. In addition, the input vector $\mathbf{u}_{\mathrm{a}}(s)$ has dimension $n \times 1$ and can be expressed as

$$
\mathbf{u}_{\mathrm{a}}(s)=\mathbf{L} \mathbf{u}(s)
$$

where $\mathbf{u}(s)$ represents the controller signals as actuator inputs, with dimension $n \times 1$. On the other hand, $\mathbf{L}$ corresponds to a diagonal control effectiveness matrix with dimension $n \times n$ :

$$
\mathbf{L}=\operatorname{diag}\left(l_{1}, l_{2}, \ldots, l_{n}\right)
$$

where the $l_{i}$ are scalars satisfying $0 \leq l_{i} \leq 1$, with $i=1,2, \ldots, n$. These scalars model the effectiveness of each particular actuator. If the $i$ th actuator is healthy, then $l_{i}=1$. In contrast, $l_{i}<1$ corresponds to a loss of effectiveness failure, and if $l_{i}=0$, then the actuator has failed completely.

For the redundancies selection procedure detailed in the following sections, the $l_{i}$ elements are parameters that can be

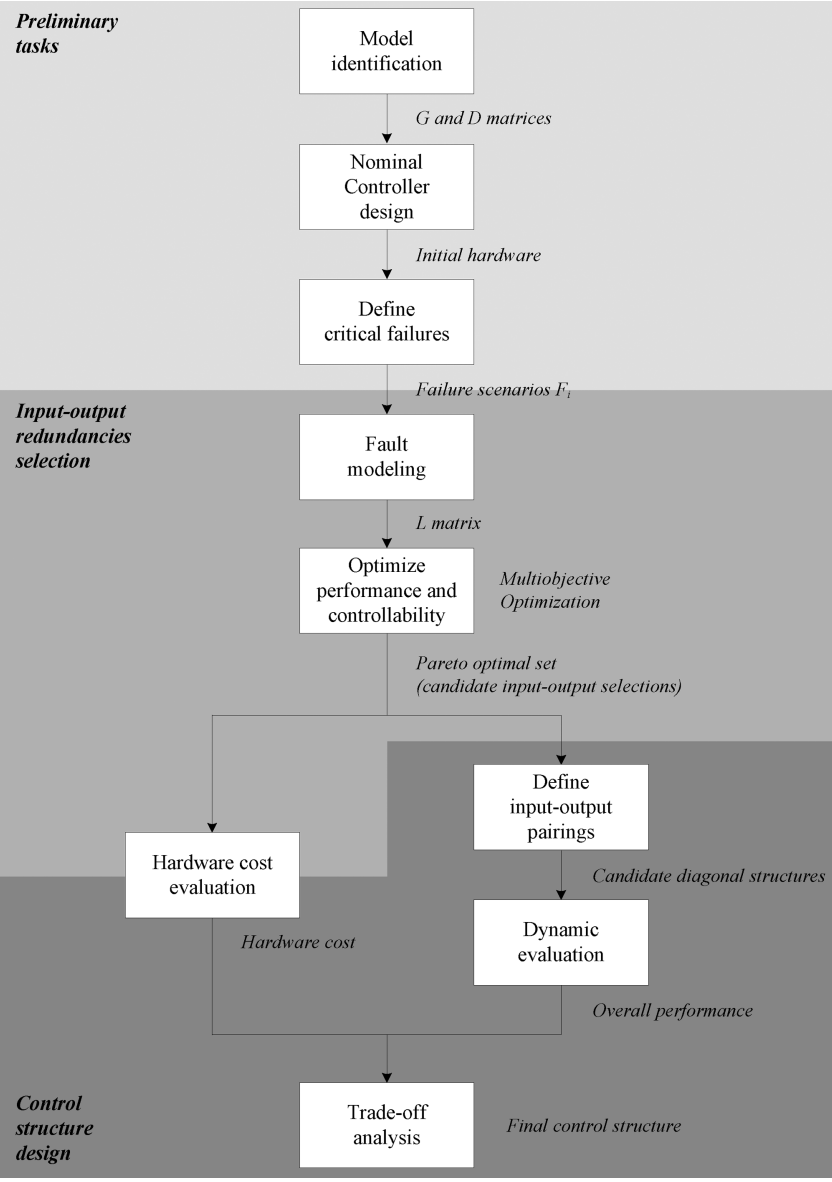

Figure 2. Overall procedure.

specified based on the considered failure cases. As an example, for a process with 8 inputs and considering a 50\% effectiveness reduction of the fifth actuator (see case 2 , section 3.1.2), then $L$ must be defined as $\mathbf{L}=\operatorname{diag}(1,1,1,1,0.5,1,1,1)$. Note that during the system operation, the necessary information to compute $\mathbf{L}$ online must be supplied by the FDD subsystem.

2.2. Performance Measure. Taking into account eqs 1 and 2 and considering disturbance variables, it can be expressed:

$$
\mathbf{y}(s)=\mathbf{G}(s) \mathbf{L u}(s)+\mathbf{D}(s) \mathbf{d}(s)
$$

where $\mathbf{D}(s)$ represents the transfer function matrix corresponding to disturbances with dimension $m \times p$. In addition, $\mathbf{d}(s)$ represents the $p \times 1$ disturbances vector. In a similar way as exposed in Zumoffen, ${ }^{10}$ eq 4 can be partitioned as

$$
\left[\begin{array}{l}
\mathbf{y}_{\mathrm{s}}(s) \\
\mathbf{y}_{\mathrm{r}}(s)
\end{array}\right]=\left[\begin{array}{ll}
\mathbf{G}_{\mathrm{s}}(s) & \mathbf{G}_{\mathrm{s}}^{*}(s) \\
\mathbf{G}_{\boldsymbol{r}}(s) & \mathbf{G}_{\mathrm{r}}^{*}(s)
\end{array}\right]\left[\begin{array}{cc}
\mathbf{L}_{\mathrm{s}} & 0 \\
0 & \mathbf{L}_{\mathrm{r}}
\end{array}\right]\left[\begin{array}{l}
\mathbf{u}_{\mathrm{s}}(s) \\
\mathbf{u}_{\mathrm{r}}(s)
\end{array}\right]+\left[\begin{array}{c}
\mathbf{D}_{\mathrm{s}}(s) \\
\mathbf{D}_{\mathrm{r}}(s)
\end{array}\right] \mathbf{d}(s)
$$

where $\mathbf{G}_{\mathrm{s}}(s)$ corresponds to the square $r \times r$ subprocess selection to be controlled, based on the selection of $r$ inputs and $r$ outputs from $\mathbf{G}(s)$. On the other hand, $\mathbf{G}_{\mathrm{s}}^{*}(s), \mathbf{G}_{\mathrm{r}}(s), \mathbf{G}_{\mathrm{r}}^{*}(s), \mathbf{D}_{s}(s)$, and $\mathbf{D}_{\mathrm{r}}(s)$ are transfer function matrices with dimension $r \times(n-r)$, $(m-r) \times r,(m-r) \times(n-r), r \times p$, and $(m-r) \times p$, respectively. Here, the effectiveness matrix $\mathbf{L}$ is partitioned as

$$
\mathbf{L}=\left[\begin{array}{cc}
\mathbf{L}_{\mathrm{s}} & 0 \\
0 & \mathbf{L}_{\mathrm{r}}
\end{array}\right]
$$


where $\mathbf{L}_{\mathrm{s}}=\operatorname{diag}\left(l_{\mathrm{s}_{1}}, l_{\mathrm{s}_{2}}, \ldots, l_{\mathrm{s}_{\mathrm{r}}}\right)$ and $\mathbf{L}_{\mathrm{r}}=\operatorname{diag}\left(l_{\mathrm{r}_{1}}, l_{\mathrm{r}_{2}}, \ldots, l_{r_{n-r}}\right)$ have dimension $r \times r$ and $(n-r) \times(n-r)$, respectively. Note that the $\mathbf{L}_{\mathrm{s}}$ elements are linked to the $r$ inputs (actuators) selected for control purposes.

From the internal model control theory (see Figure 3) and assuming steady-state perfect control $\left(y_{s}=y_{s}^{\text {set }}\right)$ then:

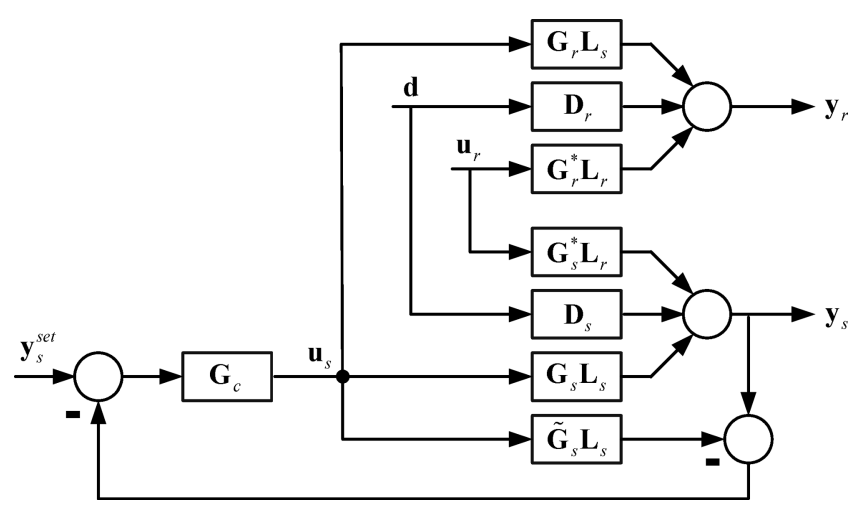

Figure 3. Internal model control structure.

$$
\begin{aligned}
\mathbf{y}_{\mathrm{r}}= & {\left[\mathbf{G}_{\mathrm{r}} \mathbf{L}_{\mathrm{s}} \mathbf{G}_{\mathrm{s}}^{-1}\right] \mathbf{y}_{\mathrm{s}}^{\text {set }}+\left[\mathbf{D}_{\mathrm{r}}-\mathbf{G}_{\mathrm{r}} \mathbf{L}_{\mathrm{s}} \mathbf{G}_{\mathrm{s}}^{-1} \mathbf{D}_{\mathrm{s}}\right] \mathbf{d} } \\
& +\left[\mathbf{G}_{\mathrm{r}}^{*} \mathbf{L}_{\mathrm{r}}-\mathbf{G}_{\mathrm{r}} \mathbf{L}_{\mathrm{s}} \mathbf{G}_{\mathrm{s}}^{-1} \mathbf{G}_{\mathrm{s}}^{*} \mathbf{L}_{\mathrm{r}}\right] \mathbf{u}_{\mathrm{r}}
\end{aligned}
$$

which shows the uncontrolled variables deviations (from their operating points) subject to set point and disturbances changes.

Within this framework, the optimal selection of $r$ inputs and $r$ outputs based on the minimization of the sum of square deviations (SSD) can be formulated as ${ }^{10}$

Problem 1

$$
\begin{aligned}
& \min _{(I, J)}\left\|\mathbf{G}_{\mathrm{r}_{(I, J)}} \mathbf{L}_{\mathrm{s}_{(I, J)}} \mathbf{G}_{\mathrm{s}_{(I, J)}}-1\right\|_{\mathrm{F}}^{2} \\
& \quad+\left\|\mathbf{D}_{\mathbf{r}_{(I, J)}}-\mathbf{G}_{\mathrm{r}_{(I, J)}} \mathbf{L}_{\mathbf{s}_{(I, J)}} \mathbf{G}_{\mathrm{s}_{(I, J)}}{ }^{-1} \mathbf{D}_{\mathbf{s}_{(I, J)}}\right\|_{\mathrm{F}}^{2}
\end{aligned}
$$

subject to

$$
\operatorname{det}\left[\mathbf{G}_{\mathbf{s}_{(I, J)}}\right] \neq 0
$$

where $I=\left\{i_{1}, i_{2}, \ldots, i_{r}\right\} \subset\{1,2, \ldots, m\}$ and $J=\left\{j_{1}, j_{2}, \ldots, j_{\mathrm{r}}\right\} \subset$ $\{1,2, \ldots, n\}$ correspond to index sets, which parametrize the selection of the controlled and manipulated variables, respectively. In addition, $\|. . .\|_{F}$ represents the Frobenius norm.

2.3. Controllability Measure. Consider a particular selection of $r$ inputs and $r$ outputs represented by $\mathbf{G}_{s}(s) \mathbf{L}_{s}$. If independent control of the $r$ outputs is required, then $\operatorname{rank}\left[\mathbf{G}_{s}(s) \mathbf{L}_{s}\right]$ $=r$ must be satisfied, that is, the selected subprocess $\mathbf{G}_{s}(s) \mathbf{L}_{\mathrm{s}}$ must be functionally controllable. ${ }^{16}$ Note that this is fulfilled if and only if $\operatorname{det}\left[\mathbf{G}_{\mathrm{s}}(s) \mathbf{L}_{\mathrm{s}}\right] \neq 0$. In contrast, $\mathbf{G}_{\mathrm{s}}(s) \mathbf{L}_{\mathrm{s}}$ is said to be functionally uncontrollable if $\operatorname{rank}\left[\mathbf{G}_{s}(s) \mathbf{L}_{s}\right]<r$.

For a functionally controllable system, it is theoretically possible to design a control strategy such that the manipulation of the control variables influences all outputs. However, this controllability criterion does not ensure acceptable control in practice. In fact, for a functionally controllable process that is ill-conditioned, some combinations of the inputs have a weak influence on the outputs, indicating control problems. ${ }^{16}$ Thus, the above functional controllability criterion is utilized in this work only as a necessary condition to discard uncontrollable input-output selections.
In order to guide the variables selection procedure, it is proposed the evaluation of the minimum singular value (MSV) at steady-state $(s=0)$, that is, $\underline{\sigma}\left[\mathbf{G}_{s} \mathbf{L}_{s}\right]$. $^{26}$ This scalar index provides a quantitative measure of the controllability of a particular $\mathbf{G}_{s} \mathbf{L}_{s}$. It can be employed to compare a set of candidate input-output selections taking into account their control effectiveness. As described in Morari, ${ }^{17}$ the MSV measures the ability to handle disturbances, plant model mismatches, etc. The larger the value of $\underline{\sigma}\left[\mathbf{G}_{s} \mathbf{L}_{s}\right]$, more likely it is to achieve acceptable control. ${ }^{26}$ Note that $\mathbf{G}_{s} \mathbf{L}_{s}$ is functionally uncontrollable if and only if $\underline{\sigma}\left[\mathbf{G}_{s} \mathbf{L}_{s}\right]=0$.

In this context, the optimal selection of $r$ inputs and $r$ outputs based on the maximization of the MSV can be formulated as

Problem 2

$$
\min _{(I, J)}-\underline{\sigma}\left[\mathbf{G}_{\mathrm{s}_{(I, J)}} \mathbf{L}_{\mathrm{s}_{(I, J)}}\right]
$$

subject to

$$
\operatorname{det}\left[\mathbf{G}_{\mathrm{s}_{(I, J)}}\right] \neq 0
$$

where the constraint of eq 11 avoids the selection of functionally uncontrollable choices, assuming that all $\mathbf{L}_{\mathrm{s}}$ elements are nonzero. Note that the set of $r+r$ selected variables consists of

1. a subset $\mathcal{S}_{1}$ consisting of input-output variables already utilized by the nominal structure, NC

2. an additional input-output subset $\mathcal{S}_{2}$ containing the redundancies

Then both subsets $\mathcal{S}_{1}$ and $\mathcal{S}_{2}$ must be considered for the design of the alternative control structures.

2.4. Multiobjective Optimization Framework. Given that the objective functions contained in eqs 8 and 10 must be optimized simultaneously, then a multiobjective optimization framework is proposed in this paper. The problem to solve is

$$
\min _{(I, J)}\left\{\operatorname{MSV}_{(I, J)}, \operatorname{SSD}_{(I, J)}\right\}
$$

subject to

$$
\operatorname{det}\left[\mathbf{G}_{\mathrm{s}_{(I, J)}}\right] \neq 0
$$

where $\operatorname{MSV}_{(I, J)}$ and $\operatorname{SSD}_{(I, J)}$ refers to the functional costs contained in eqs 10 and 8 , respectively. The nondominated solutions of eq 12 conform the so-called Pareto optimal set, $\mathcal{P}$, where any pair of solutions $(I, J)_{i},(I, J)_{j} \in \mathcal{P}$ satisfies

$$
\operatorname{MSV}_{(I, J)_{i}}<\operatorname{MSV}_{(I, J)_{j}} \text { and } \operatorname{SSD}_{(I, J)_{i}}>\operatorname{SSD}_{(I, J)_{j}}
$$

As shown in section 3, these objective functions are conflicting because there is no solution that optimizes both criteria. In the literature, several methodologies propose to transform the problem to a single-objective optimization framework. However, it is complicated to aggregate the value of different objective functions in the context of control structure selection based on multiobjective optimization. ${ }^{13,27}$

In this work, the Pareto optimal set corresponding to the problem of eq 12 is obtained by using the Matlab function gamultiobj, ${ }^{28}$ which implements a multiobjective controlled elitist genetic algorithm (GA). As the algorithm progresses, it generates solutions that ${ }^{15}$ (i) minimize the values of the functional costs and (ii) help to increase the diversity in order to converge to the Pareto front. In this paper, a vectorized version of the objective functions is implemented so as to speed up the algorithm. 
Concerning the GA, it is a global optimization procedure that imitates natural biological dynamics. Some of its interesting features include (i) suitability for solving large combinatorial problems and (ii) presenting low likelihood of obtaining local optima. Note that through this procedure, the large set of feasible solutions corresponding to the variables selection problem is reduced to analyzing only a small set of Pareto optimal solutions.

Once the Pareto optimal set is available, the next step is to select the final solution. Usually, an expert on the subject must define the most preferred solution according to some particular criterion. ${ }^{29}$ In the following section, a cost-based approach is proposed to support this task. Anyway, the chosen solution must behave satisfactorily when it is implemented and tested through dynamic simulations (see section 3).

2.5. Hardware Cost. As stated above, the input-output selection is mainly based on the optimization of controllability and performance indexes. The obtained Pareto optimal solutions generally involve existing hardware from the nominal structure NC, as well as extra actuators and sensors (redundancies), which obviously imply an additional cost. In this work, the objective is to establish a low-cost redundancies set in order to design efficient control structures.

In the context of GA, consider the following search chromosome, which represents a particular hardware selection:

$$
\boldsymbol{C}_{\boldsymbol{i}}=\left[c_{1}, c_{2}, \ldots, c_{N_{\mathrm{c}}}\right]
$$

Note that $C_{i}$ has length $N_{\mathrm{c}}=m+n$, that is, $m$ decision variables for the CVs and $n$ for the MVs. In addition, each particular gene $c_{i}$ of $C_{i}$ with $i=1,2, \ldots, N_{\mathrm{c}}$ belongs to a binary alphabet where $c_{i}=1$ indicates the utilization of the sensor/actuator corresponding to the $i$ location, and $c_{i}=0$ implies the opposite situation. For a particular solution $C_{i}$, the corresponding hardware cost, $C_{\mathrm{hw}}$, can be calculated as

$$
C_{\mathrm{hw}}=\boldsymbol{C}_{i} \boldsymbol{C}^{T}
$$

where $C$ corresponds to

$$
\mathbf{C}=\left[c_{1}^{*}, c_{2}^{*}, \ldots, c_{N_{\mathrm{c}}}^{*}\right]
$$

Each element $c_{i}^{*}$ represents the cost associated with the use of each particular hardware component. Typically, $c_{i}^{*}=0$ can be defined for the original hardware contained in the NC structure. Thus, a nonzero $C_{\mathrm{hw}}$ indicates that the solution $C_{i}$ recommends the utilization of some redundancies.

2.6. Control Structure Design. For simplicity and in line with a previous work, ${ }^{7}$ this paper proposes the implementation of decentralized control strategies, which can be synthesized through single-input/single-output (SISO) PID controllers. However, more complex structures can be considered to improve system performance. $^{10}$

In this context, the next step of the procedure consists of the input-output pairing task to define the $\mathrm{MV}-\mathrm{CV}$ interconnections in order to implement the SISO PID controllers. The pairing problem is solved here based on a branch and bound solution proposed by Kariwala and $\mathrm{CaO}^{22}$ which employs a biobjective selection criteria involving the RGA sum and the $\mu$ interaction measure.

The complete methodology can be summarized as (see Figure 2):

1. Specify the effectiveness matrix $\mathbf{L}$ taking into account the failures set to be managed.

2. Execute the multiobjective GA to optimize the performance and controllability indexes detailed in sections 2.2 and 2.3 As a result, the Pareto front is obtained at the objective plane (SSD vs MSV).

3. For each solution from step 2

- Calculate the corresponding hardware cost (section 2.5).

- Execute the branch and bound algorithm to define the input-output pairings.

- Implement and evaluate the behavior of the control structure through dynamic simulations. This task is detailed in section 3 .

4. From step 3, select the final control structure by trading-off the overall performance and the hardware cost.

\section{CASE STUDY: TENNESSEE EASTMAN PROCESS}

The well-known Tennessee Eastman case study is considered here to comprehensively evaluate the methodology presented in the previous section. Note that this paper provides minimal information about the TE process. For more details, please refer to Downs and Vogel. ${ }^{23}$

3.1. Redundancies selection and controller design. For this stage, the nominal controller (NC) proposed by Luppi et al. is adopted. It is a fully decentralized control structure of reduced dimension, formed by four independent SISO control loops (without considering the stability loops). Table 1 summarizes the

\section{Table 1. Nominal Controller Control Loops}

\begin{tabular}{cccr} 
MV & $\mathrm{CV}$ & $K_{\mathrm{c}}$ & $T_{\mathrm{i}}$ \\
$\mathrm{xmv}(1)$ & $\mathrm{xme}_{\mathrm{G} / \mathrm{H}}$ & 9.5 & 1 \\
$\operatorname{xmv}(3)$ & $\mathrm{xme}(7)$ & -0.2 & 10 \\
$\operatorname{xmv}(4)$ & $\mathrm{xme}(17)$ & 1.5 & 1 \\
$\operatorname{xmv}(6)$ & $\mathrm{xme}(30)$ & -2.7 & 2 \\
\hline
\end{tabular}

$\mathrm{CV}-\mathrm{MV}$ pairings, as well as the tuning parameters, of each PI controller. Note that the NC employs a reduced number of CVs and MVs. Thus, all the remaining sensors and actuators that are not utilized by NC can be considered for the redundancies selection problem and the alternative control structure design. These variables are detailed in Table 2.

\begin{tabular}{|c|c|c|c|}
\hline output & description & input & description \\
\hline $\operatorname{xme}(5)$ & $\begin{array}{l}\text { recycle flow } \\
\text { (stream 8) }\end{array}$ & $\operatorname{xmv}(5)$ & compressor recycle valve \\
\hline$x m e(6)$ & $\begin{array}{l}\text { reactor feed rate } \\
\quad(\text { stream } 6)\end{array}$ & $\operatorname{xmv}(9)$ & stripper steam valve \\
\hline xme(9) & reactor temp & $\mathrm{xmv}(11)$ & condenser cooling water flow \\
\hline $\mathrm{xme}(11)$ & $\begin{array}{l}\text { product separator } \\
\text { temp }\end{array}$ & $\operatorname{xme}(21) s p$ & $\begin{array}{l}\text { reactor cooling water outlet } \\
\text { temp set point }\end{array}$ \\
\hline xme (13) & $\begin{array}{l}\text { product separator } \\
\text { pressure }\end{array}$ & & \\
\hline xme (16) & stripper pressure & & \\
\hline xme(18) & stripper temp & & \\
\hline $\operatorname{xme}(20)$ & compressor work & & \\
\hline
\end{tabular}

Table 2. Redundancies Selection Problem: Available Variables

Prior to these tasks, a set of critical failures was determined through several closed-loop simulations of the rigorous TE process model. ${ }^{23}$ Here, the term critical means that if certain corrective actions are not taken by the system, then a shutdown of the process will occur. In this work, these actions involve the reconfiguration of the $\mathrm{NC}$ structure since a readjustment of the nominal PI controllers is insufficient. Table 6 in Downs and 
Vogel $^{23}$ lists some process operating constraints that must be respected for equipment protection, including high and low shutdown limits. In the following sections, four cases are analyzed, and the corresponding faults are presented in Table 3.

\section{Table 3. Proposed Faults}

\begin{tabular}{cll} 
fault & \multicolumn{1}{c}{ description } & \multicolumn{1}{c}{ type } \\
$F_{1}$ & $20 \%$ reduction of effectiveness of actuator $\mathrm{xmv}(3)$ & partial failure \\
$F_{2}$ & $50 \%$ reduction of effectiveness of actuator $\mathrm{xmv}(6)$ & partial failure \\
$F_{3}$ & blockade of actuator $\mathrm{xmv}(6)$ & total failure \\
$F_{4}$ & blockade of actuators $\mathrm{xmv}(1), \operatorname{xmv}(4), \operatorname{xmv}(6)$ & simultaneous failures
\end{tabular}

Taking into account these failure scenarios, some general considerations concerning the redundancies selection problem and control structure design include:

- The process operation mode 1 (base case) is considered for this study, with a $\mathrm{G} / \mathrm{H}$ mass ratio of $50 / 50$ and a production rate of $7038 \mathrm{~kg} \mathrm{~h}^{-1} \mathrm{G}$ and $7038 \mathrm{~kg} \mathrm{~h}^{-1} \mathrm{H}^{23}$ The corresponding (normalized) steady-state model, that is, the matrices $\mathbf{G}$ and $\mathbf{D}$ are the same as used in Luppi et al. $^{7}$

- As a simplifying assumption, $r=4$ (the dimension of the control structure) was initially selected for all design cases. However, $r=5,6$, etc., can be chosen to try to improve the system performance (see section 3.1.2).

- In previous works, ${ }^{7,10}$ the selection of xme(7), xme(17), $\mathrm{xme}(30)$ and $\mathrm{xme}_{\mathrm{G} / \mathrm{H}}$ as controlled variables was forced to comply with all TE process requirements. Here, this restriction is relaxed since some performance degradation is tolerated due to faults. This hypothesis is valid only if all the operating constraints are not violated (to protect the equipment). Anyway, the selection of $x m e(7)$, xme(17), $\mathrm{xme}(30)$, and $\mathrm{xme}_{\mathrm{G} / \mathrm{H}}$ is enforced through the following cost vector.

- The cost vector (eq 17) is defined as $C=[10,10,10,10$, $10,10,10,10,0,0,0,0,0,0,0,10,0,10,10,10]$. Note that the null elements of $C$, i.e. $C(9,10,11,12,13,14,15,17)$ correspond to the original hardware employed by $\mathrm{NC}$, that is, $x m e(7), x m e(17), x m e(30), x e_{G / H}, x m v(1), x m v(3)$, $x m v(4)$, and $x m v(6)$, respectively. Thus, when any additional sensor or actuator is selected, the cost is incremented by 10 units.

- For each GA execution, the utilized main parameters were number of variables $=20$, population type $=$ bit string, population size $=10000$, tournament size $=2$, crossover fraction $=0.8$, mutation rate $=0.01$, migration fraction $=$ 0.5 , migration interval $=1$, number of generations $=200$, and user function evaluation $=$ vectorized.

Four different fault scenarios are presented here to demonstrate the potentiality of the methodology. The corresponding dynamic analysis is detailed in section 3.2.

3.1.1. Case 1: Partial Failure of Actuator $x m v(3)\left(F_{1}\right)$. For this case, a $20 \%$ effectiveness reduction of actuator $\mathrm{xmv}(3)$ is considered, called $F_{1}$. From closed-loop simulation of $\mathrm{NC}$ with $F_{1}$, a process shutdown was detected after the occurrence of $\operatorname{idv}(1)$ due to a very high reactor pressure. By analyzing $\mathrm{NC}$, it is noted that the original tuning of the loop $x m v(3)-x m e(7)$ is too aggressive. If its static gain is increased trying to counteract the effect of $F_{1}$, this would lead to very underdamped process dynamics. In contrast, a smoother tuning of $x m v(3)-x m e(7)$ for NC does not allow the rejection of $\operatorname{idv}(1)$.

Then, taking into account $F_{1}$ and $\operatorname{idv}(1)$, the GA was executed to solve the problem stated in eq 12. The obtained Pareto front is presented in Figure 4a, corresponding to a SSD vs - MSV graph. In order to select the final control structure, the following general criterion was utilized:

1. From the obtained Pareto front, select the lowest cost solution

2. From step 1 , if there are multiple equal-cost alternatives, then select the solution with the highest MSV

After this analysis, only seven alternatives from the Pareto optimal set can be distinguished (from a total of 17 solutions, see Figure $4 \mathrm{a})$. They represent low-cost designs ( cost $=20$ or 30 ) when compared against the remaining solutions (cost $=40,50$, $60,70)$. Through dynamic simulation, it was determined that only two of these seven alternatives are able to handle $F_{1}$ avoiding
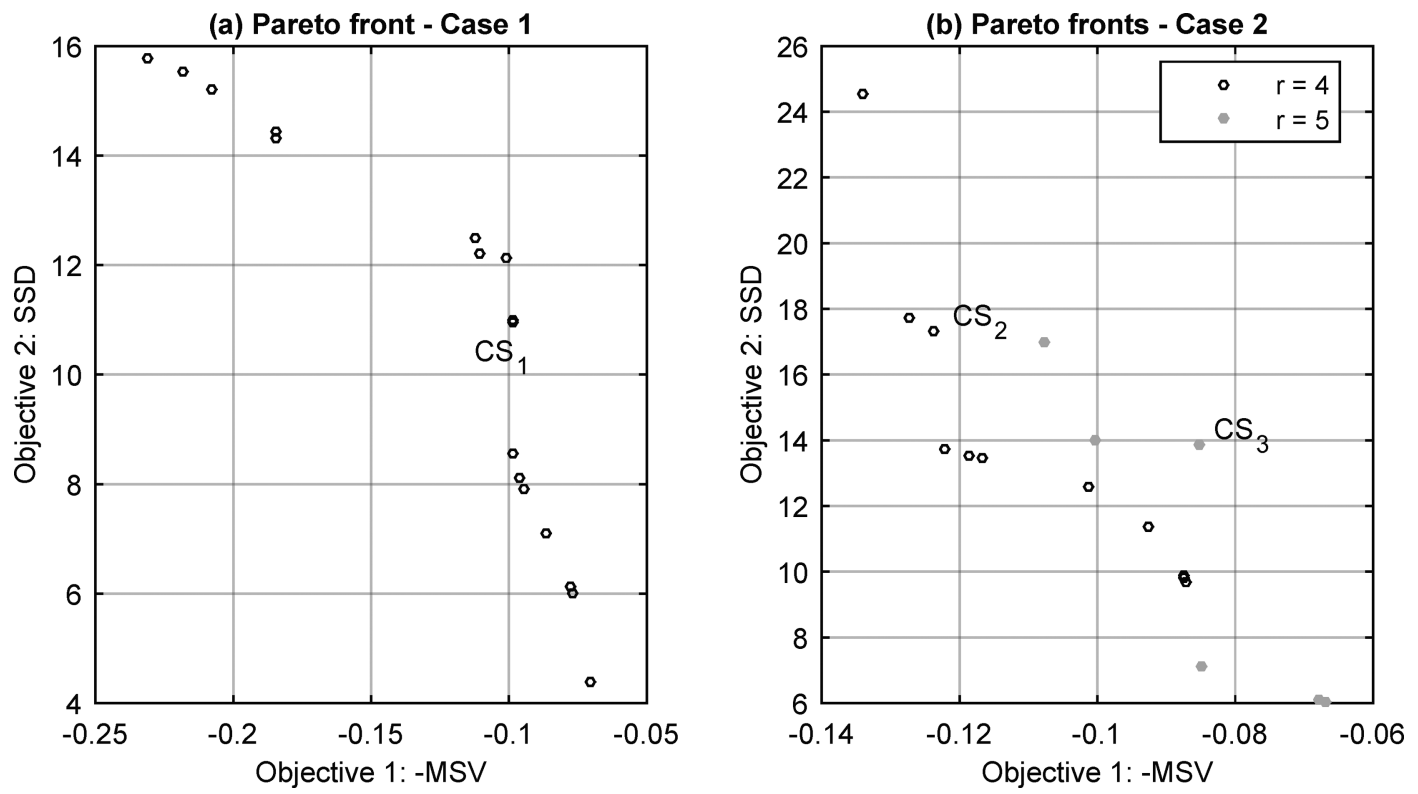

Figure 4. Alternative solutions: (a) fault $F_{1}$ (case 1); (b) fault $F_{2}$ (case 2). 
a shutdown of the process. These solutions correspond to structures that maintain the selection of $x m v(3)$. Since both alternatives have cost $=30$, then the solution with higher MSV (named as $\mathrm{CS}_{1}$ ) was taken, which is detailed in Table 4. Here, the selected redundancies are two sensors (xme(9) and xme(20)) and xme(21)sp, which is not a physical actuator. Note that the implementation of $\mathrm{CS}_{1}$ implies a minor reconfiguration of $\mathrm{NC}$, which is reflected in the associated low cost. While the tuning of the loop $x m v(3)-x m e(7)$ is much more robust within the framework of $\mathrm{CS}_{1}$, one disadvantage is that this design still employs the failed actuator $\mathrm{xmv}(3)$. A complete analysis of the dynamic performance of $\mathrm{CS}_{1}$ is presented in section 3.2.

3.1.2. Case 2: Partial Failure of Actuator xmv $(6)\left(F_{2}\right)$. Here, a $50 \%$ effectiveness reduction of actuator $\operatorname{xmv}(6)$ is analyzed (called $F_{2}$ ). As indicated by the closed-loop simulation of NC with $F_{2}$, a shutdown of the process is produced when disturbances $\operatorname{idv}(1)$ and $\operatorname{idv}(2)$ are considered together. For this scenario $\left(F_{2}, \operatorname{idv}(1)\right.$ and $\left.\operatorname{idv}(2)\right)$, two alternative designs were proposed considering $r=4$ and $r=5$, where $r$ corresponds to the control structure dimension. The aim is system performance improvement by using five control loops instead of four. The GA was executed twice in order to find solutions for each case. The obtained solutions are shown in Figure $4 \mathrm{~b}$, where the different solution sets corresponding to $r=4$ and $r=5$ are overlapped. The obtained Pareto optimal set consists of 12 solutions for $r=4$ and 6 solutions for $r=5$. For $r=4$, the solution $\mathrm{CS}_{2}$ presented in Table 4 was chosen because (i) it has the lowest cost ( cost $=30)$ and the highest MSV and (ii) it does not include $\operatorname{xmv}(6)$, being able to overcome any value of effectiveness reduction of this actuator. For this case, the selected redundancies are two sensors (xme(18) and xme(20)) and one actuator, $x m v(5)$. However, as will be shown in section $3.2, \mathrm{CS}_{2}$ has poor performance in relation to the $r=5$ solution (called $\mathrm{CS}_{3}$ ). This is consistent with the higher SSD value presented by $\mathrm{CS}_{2}$, see Table 4. On the other hand, for $r=5, \mathrm{CS}_{3}$ was finally selected. For this case, the selected redundancies were two sensors (xme(9) and xme(20)) and one actuator, $x m v(11)$. This structure presents the same cost as $\mathrm{CS}_{2}$ but improves the system performance. On the downside, $\mathrm{CS}_{3}$ utilizes the failed actuator $\mathrm{xmv}(6)$. In section 3.2, the dynamic performance of $\mathrm{CS}_{2}$ and $\mathrm{CS}_{3}$ is compared.

3.1.3. Case 3: Total Failure of Actuator xmv(6) $\left(F_{3}\right)$. In this case, a blockade of actuator $\mathrm{xmv}(6)$ is considered, called $F_{3}$. From closed-loop simulation of $\mathrm{NC}$ with $F_{3}$, a process shutdown was detected after the occurrence of $\operatorname{idv}(1)$ and $\operatorname{idv}(2)$ due to a high reactor pressure. Taking into account $F_{3}, \operatorname{idv}(1)$, and $\operatorname{idv}(2)$, the GA was executed to solve the problem stated in eq 12 . The obtained solutions are presented in Figure 5a, where 10 solutions compose the Pareto optimal set. Here, the lowest cost solution coincides with $\mathrm{CS}_{2}$ obtained previously (section 3.1.2). For this case, it is proposed to examine whether it is possible to find a cheaper design (i.e., with cost $<30$ ). To this end, a singleobjective GA was executed to directly minimize the hardware cost expressed in eq 16. The best solutions are presented in Figure 5a, overlapped with those obtained previously. Note the shifting of the current solutions set with respect to the previous

Table 4. Alternative Control Structures for the Considered Faults $F_{i}$

\begin{tabular}{|c|c|c|c|c|c|}
\hline & $\mathrm{CS}_{1}\left(F_{1}\right)$ & $\mathrm{CS}_{2}\left(F_{2}\right)$ & $\mathrm{CS}_{3}\left(F_{2}\right)$ & $\mathrm{CS}_{4}\left(F_{3}\right)$ & $\mathrm{CS}_{5}\left(F_{4}\right)$ \\
\hline & $\begin{array}{l}x m v(1)-x^{-} e_{G / H} \\
x m v(3)-x m e(7) \\
x m v(4)-x m e(9) \\
x m e(21) \operatorname{sp}-x m e(20)\end{array}$ & $\begin{array}{l}x m v(1)-x_{G} e_{G / H} \\
x m v(3)-x m e(7) \\
x m v(4)-x m e(18) \\
x m v(5)-x m e(20)\end{array}$ & $\begin{array}{l}x m v(1)-x e_{G / H} \\
x m v(3)-x m e(7) \\
x m v(4)-x m e(9) \\
x m v(6)-x m e(30) \\
x m v(11)-x m e(20)\end{array}$ & $\begin{array}{l}x m v(1)-x_{G} e_{G / H} \\
x m v(3)-x m e(7) \\
x m v(4)-x m e(17) \\
x m v(5)-x m e(20)\end{array}$ & $\begin{array}{l}\operatorname{xmv}(3)-x m e(7) \\
\operatorname{xmv}(5)-x m e(20) \\
\operatorname{xmv}(11)-x m e(5) \\
x m e(21) \operatorname{sp}-x m e(11)\end{array}$ \\
\hline$-\mathrm{MSV}$ & -0.099 & -0.124 & -0.085 & -0.123 & -0.076 \\
\hline SSD & 10.97 & 17.30 & 13.87 & 17.66 & 11.28 \\
\hline cost & 30 & 30 & 30 & 20 & 60 \\
\hline
\end{tabular}

(a) Case 3

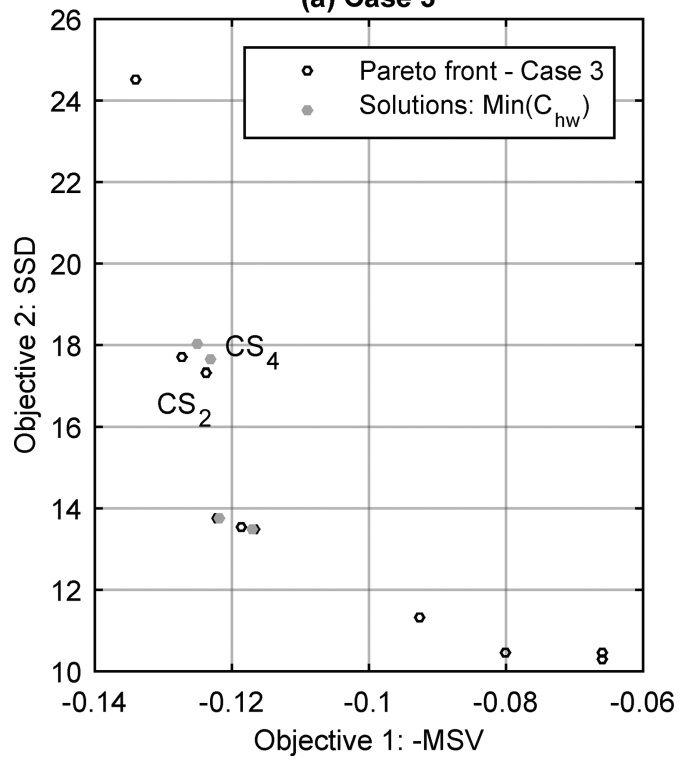

(b) Pareto front - Case 4

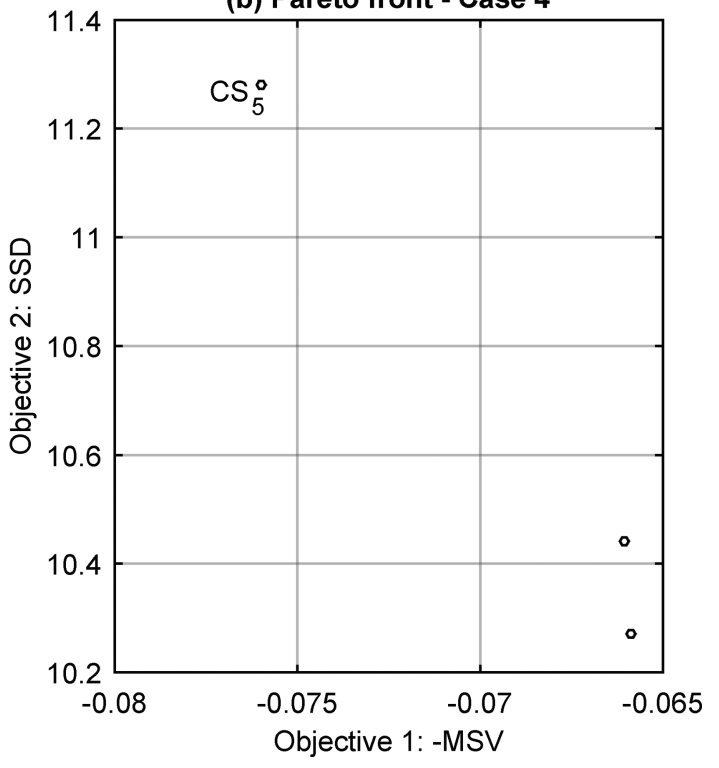

Figure 5. Alternative solutions: (a) fault $F_{3}$ (case 3); (b) fault $F_{4}$ (case 4). 
Table 5. Simulation Parameters

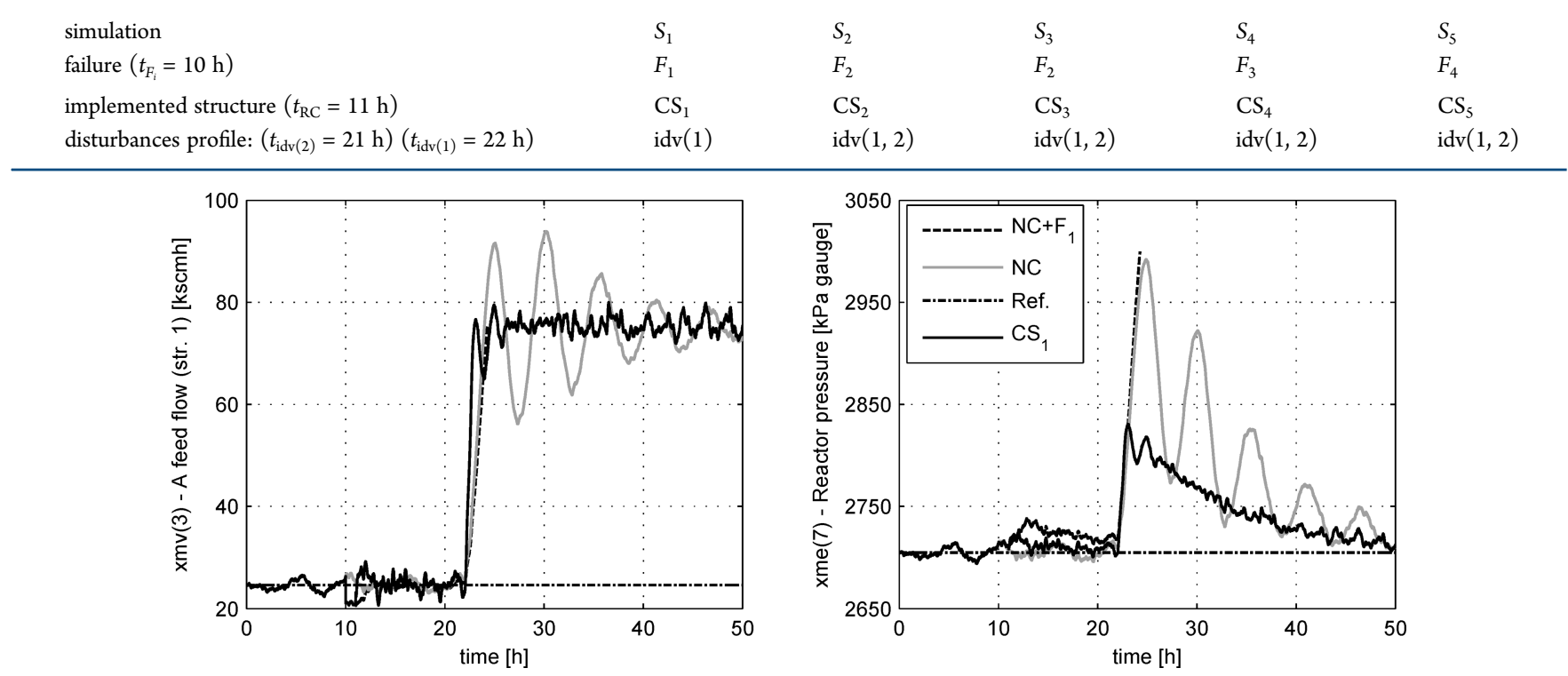

Figure 6. Simulation $S_{1}, \mathrm{xmv}(3)$ and $\mathrm{xme}(7)$ dynamic responses.

\section{Table 6. Dynamic Performance Comparison}

\begin{tabular}{|c|c|c|c|c|c|c|c|}
\hline index & IAE & IAE & EIP [\%] & EIP $[\%]$ & EIP $[\%]$ & $\operatorname{EIP}[\%]$ & EIP [\%] \\
\hline structure(s) & $\mathrm{NC}$ & $\mathrm{NC}$ & $\mathrm{CS}_{1}, \mathrm{NC}$ & $\mathrm{CS}_{2}, \mathrm{NC}$ & $\mathrm{CS}_{3}, \mathrm{CS}_{2}$ & $\mathrm{CS}_{4}, \mathrm{NC}$ & $\mathrm{CS}_{5}, \mathrm{NC}$ \\
\hline dist./simulation & $\operatorname{idv}(1)$ & $\operatorname{idv}(1,2)$ & $S_{1}$ & $S_{2}$ & $S_{3}$ & $S_{4}$ & $S_{5}$ \\
\hline xme(1) & $3.97 \times 10^{3}$ & $4.14 \times 10^{3}$ & -0.92 & -9.85 & 9.24 & 0.71 & -10.01 \\
\hline$x m e(2)$ & $2.38 \times 10^{5}$ & $2.19 \times 10^{5}$ & 16.94 & -312.74 & 41.19 & 7.64 & -16.49 \\
\hline xme(3) & $1.48 \times 10^{6}$ & $1.65 \times 10^{6}$ & 21.83 & -12.34 & 11.06 & 23.36 & -214.74 \\
\hline xme(4) & $4.08 \times 10^{3}$ & $2.45 \times 10^{3}$ & 6.92 & 5.10 & -1.64 & -59.07 & 83.44 \\
\hline $\mathrm{xme}(7)$ & $2.71 \times 10^{5}$ & $2.79 \times 10^{5}$ & 44.10 & -588.30 & 9.17 & -597.47 & -648.08 \\
\hline $\mathrm{xme}(17)$ & $1.44 \times 10^{3}$ & $1.30 \times 10^{3}$ & 15.99 & -373.64 & 44.58 & 13.28 & -845.18 \\
\hline $\mathrm{xme}(30)$ & $1.68 \times 10^{3}$ & $4.21 \times 10^{3}$ & -192.65 & -2031.00 & 38.53 & -1535.80 & -1826.37 \\
\hline $\mathrm{xme}_{\mathrm{G} / \mathrm{H}}$ & $2.37 \times 10^{2}$ & $2.05 \times 10^{2}$ & 36.74 & 9.15 & 12.12 & 13.63 & -501.20 \\
\hline Op. costs & $1.66 \times 10^{6}$ & $2.49 \times 10^{6}$ & -3.05 & 34.07 & -11.91 & 31.58 & 32.02 \\
\hline
\end{tabular}

one. This is because the minimization did not focus on SSD and MSV. The final solution $\mathrm{CS}_{4}$ has a cost of 20 and is detailed in Table 4. The commissioning of $\mathrm{CS}_{4}$ implies the implementation of only one new control loop: $\operatorname{xmv}(5)-x m e(20)$. Thus, the selected redundancies are one sensor $(x m e(20))$ and one actuator $(\operatorname{xmv}(5))$. This reconfiguration of NC allows it to handle several failure scenarios related to the actuator $x m v(6)$, including partial and total failures. A complete analysis of the dynamic performance of $\mathrm{CS}_{4}$ is presented in section 3.2.

From the results, it can be concluded that the use of an oversized set of redundancies (higher hardware cost) does not imply an improved system fault-tolerant capability. Similar conclusions were exposed in Nieto Degliuomini et al. ${ }^{21}$ and Zumoffen $^{10}$ in the context of FDD systems and plantwide control design, respectively.

3.1.4. Case 4: Total Failure of Actuators xmv(1), xmv(4), and $x m v(6)\left(F_{4}\right)$. Here, a simultaneous blockade of actuators $\operatorname{xmv}(1), \operatorname{xmv}(4)$, and $\operatorname{xmv}(6)$ is analyzed (called $\left.F_{4}\right)$. As indicated by the closed-loop simulation of $\mathrm{NC}$ with $F_{4}$, a shutdown of the process is produced when disturbances $\operatorname{idv}(1)$ and $\operatorname{idv}(2)$ are acting together. This case represents a very complicated scenario. The actuators $\mathrm{xmv}(1)$ and $\mathrm{xmv}(4)$ (which were selected in all previous designs) cannot be utilized here. For this scenario $\left(F_{4}, \operatorname{idv}(1)\right.$, and $\left.\operatorname{idv}(2)\right)$, the GA was executed to find solutions for the problem stated in eq 12. The obtained solutions are shown in Figure 5b, where the Pareto optimal set presents three alternative solutions. For this case, the solution $\mathrm{CS}_{5}$ was chosen because it possesses the lowest cost $($ cost $=$ $60)$ and the highest MSV. The selected redundancies are three sensors (xme(5), xme(11), and $\mathrm{xme}(20))$ and two actuators $(x m v(5)$ and $x m v(11))$, because $x m e(21)$ sp does not represent a physical actuator. Note that for $\mathrm{CS}_{5}$ two pairing options are possible: (i) $x m v(3)-x m e(7), x m v(5)-x m e(20), x m v(11)-x m e(11)$, $\mathrm{xme}(21) \mathrm{sp}-\mathrm{xme}(5)$ or (ii) $\mathrm{xmv}(3)-\mathrm{xme}(7), \mathrm{xmv}(5)-\mathrm{xme}(20)$, $x m v(11)-x m e(5), x m e(21) s p-x m e(11)$. The selected solution corresponds to the lowest RGA number, ${ }^{16}$ and it is detailed in Table 4. The downside is that the implementation of $\mathrm{CS}_{5}$ implies a major reconfiguration of $\mathrm{NC}$, which is reflected in its high cost. In section 3.2, the dynamic performance of $\mathrm{CS}_{5}$ is evaluated.

3.2. Dynamic Performance Analysis. This section describes the execution of several simulations of the FTC system. The objective is to compare the dynamic performance of the nominal controller developed in Luppi et al. ${ }^{7}$ against the designed fault-tolerant system, subject to several failure scenarios. In order to simulate the reconfiguration of the system (i.e., the implementation of a particular $\mathrm{CS}_{i}$ from $\mathrm{NC}$ ), the following chronological sequence of events was proposed:

1. Initially, fault-free operation of the process is considered. It is controlled with NC. 

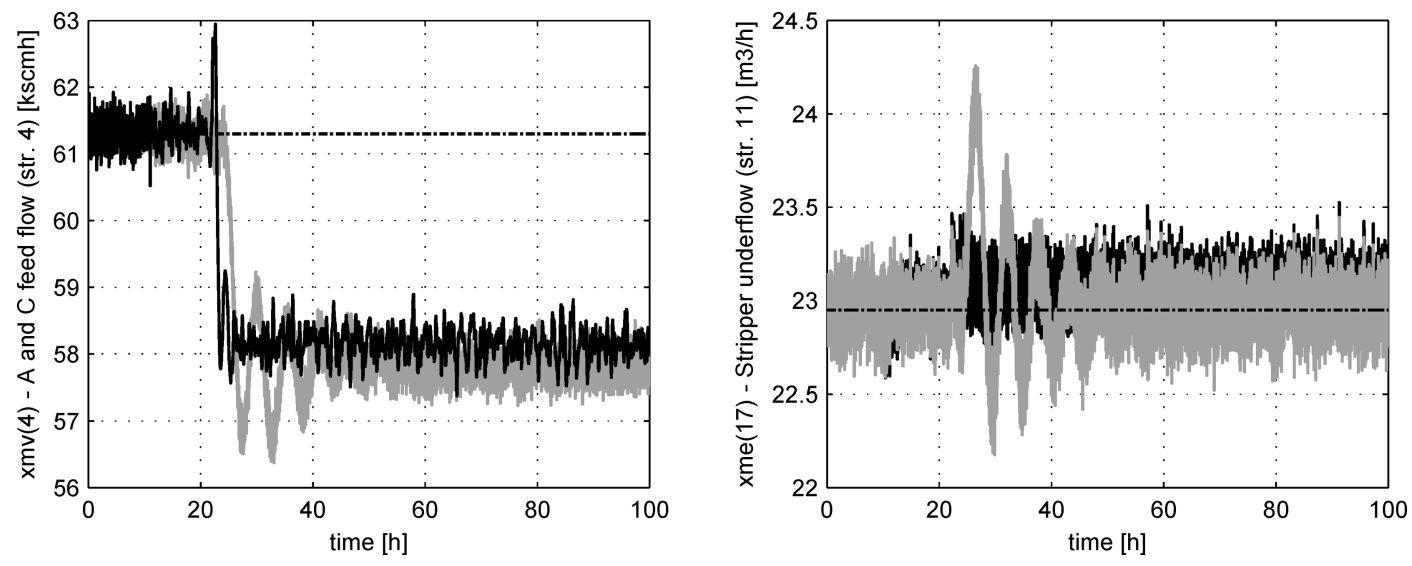

Figure 7. Simulation $S_{1}, \mathrm{xmv}(4)$ and $\mathrm{xme}(17)$ dynamic responses.
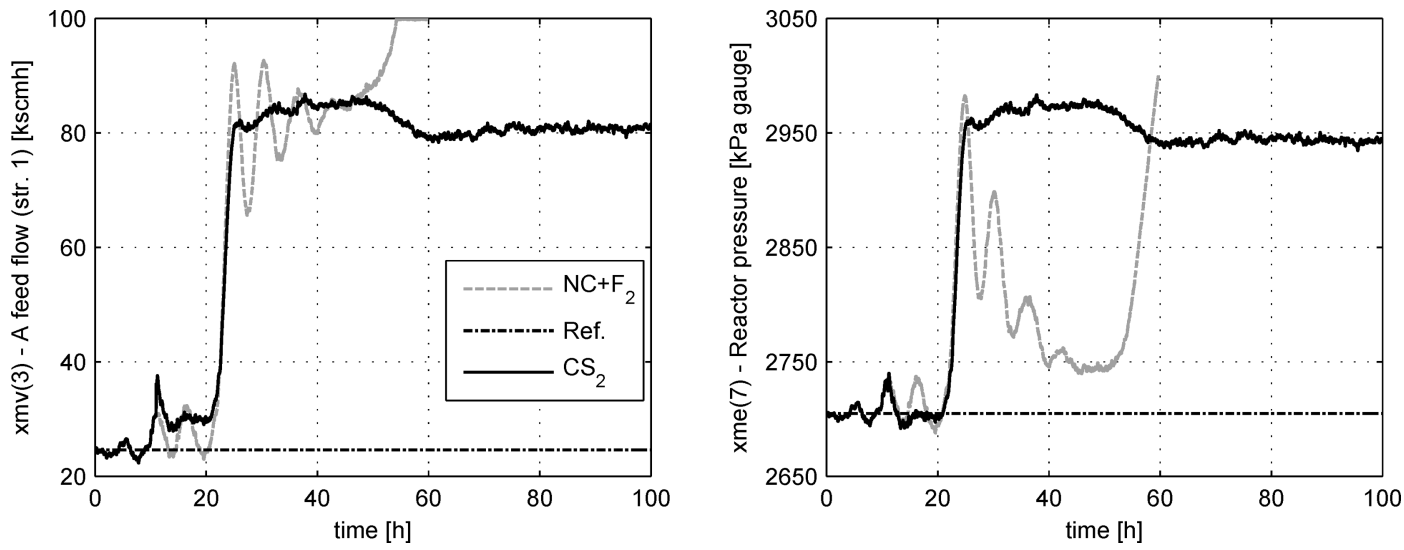

Figure 8. Simulation $S_{2}, \operatorname{xmv}(3)$ and $x m e(7)$ dynamic responses.

2. A fault $F_{i}$ occurs at $t_{F_{i}}$, and the fault detection and diagnosis (FDD) period begins. It extends until the system is reconfigured at $t_{\mathrm{RC}}$, that is, $t_{\mathrm{RC}}=t_{\mathrm{F}_{i}}+t_{\mathrm{FDD}}$. Between $t_{\mathrm{F}_{i}}$ and $t_{\mathrm{RC}}$, the process is still controlled by NC.

3. At $t_{\mathrm{RC}}$, the NC structure is reconfigured and $\mathrm{CS}_{i}$ is implemented.

4. A disturbance $\operatorname{idv}(i)$ is applied to the system at $t_{\mathrm{idv}(\mathrm{i})}$.

For all the tests, it is assumed that the fault estimation time is $t_{\mathrm{FDD}}>30 \tau_{\min }$, where $\tau_{\min }$ corresponds to the smallest time constant of the process. ${ }^{4}$ For the TE process, it is considered that $\tau_{\min }=0.01 \mathrm{~h}^{23}$ Thereby $t_{\mathrm{FDD}}=1 \mathrm{~h}$ is adopted. For each simulation, $S_{i}$, the utilized parameters are specified in Table 5 .

Figures 6-15 show the temporal responses of significant process inputs and outputs. As requested in Downs and Vogel, ${ }^{23}$ Table 6 presents a quantitative comparison based on the evaluation of the integral absolute error (IAE) and the error improvement percent (EIP): ${ }^{23}$

$$
\begin{gathered}
\mathrm{IAE}=\int_{t_{1}}^{t_{2}}|r(t)-y(t)| \mathrm{d} t \text { and } \\
\mathrm{EIP}=\frac{\mathrm{IAE}^{\text {base }}-\mathrm{IAE}^{\text {new }}}{\mathrm{IAE}^{\text {base }}} 100
\end{gathered}
$$

where $r(t)$ represents the set point, $y(t)$ represents the process output, $\left[t_{1}, t_{2}\right]$ represents the evaluation period, base is the control structure considered as reference, and new is an alternative solution to be evaluated.
All the implemented control structures $\mathrm{CS}_{i}$ are based on independent SISO PI controllers. Their corresponding $K_{\mathrm{c}}$ and $\tau_{\mathrm{I}}$ parameters are detailed in Table S1 (appendix A). In addition, a simple reconfiguration mechanism that relies on switching the operation modes of the PI controllers (automatic/manual) is employed. The aim is to minimize the introduction of meaningful transients during the commutation of the controllers.

3.2.1. Dynamic Behavior. Concerning simulation $S_{1}$, Figure 6 displays the temporal evolution of the $\mathrm{xmv}(3)-\mathrm{xme}(7)$ control loop. As commented in section 3.1.1, the NC structure presents an aggressive dynamic behavior in the presence of $\operatorname{idv}(1)$. In contrast, $\mathrm{CS}_{1}$ exhibits good regulatory performance $(\operatorname{EIP}(x m e(7))=44.10 \%)$ without excessive energy requirements since $E_{\mathrm{u}}(\mathrm{xmv}(3))$ (i.e., the control energy expenditure) is very similar for both control structures. In addition, Figure 6 shows the shutdown of the process after the occurrence of $F_{1}(t=10 \mathrm{~h})$ and $\operatorname{idv}(1)(t=22 \mathrm{~h})$. This is due to the violation of the reactor pressure (xme (7)) high limit of $3000 \mathrm{kPa}$.

Figure 7 shows the A and C feed flow (stream 4) $(x m v(4))$ and the stripper underflow (stream 11) (xme(17)) responses, respectively. The $\mathrm{CS}_{1}$ structure denotes acceptable performance with regard to NC. In fact, for most outputs, a positive EIP value can be seen, as presented in Table 6. In particular, EIP $=15.99 \%$ for $x m e(17)$ (see Figure $7 \mathrm{~b}$ ). In this case, $\mathrm{CS}_{1}$ is more effective for rejecting $\operatorname{idv}(1)$, but it presents a larger offset since xme(17) is not controlled with the $\mathrm{CS}_{1}$ structure. Figure $7 \mathrm{a}$ shows an example of the effect of the $\mathrm{NC}$ reconfiguration on $\operatorname{xmv}(4)$ dynamics: at $t=11 \mathrm{~h}$, the system stops controlling xme(17) and begins to control xme(9). Note the smooth dynamics of 

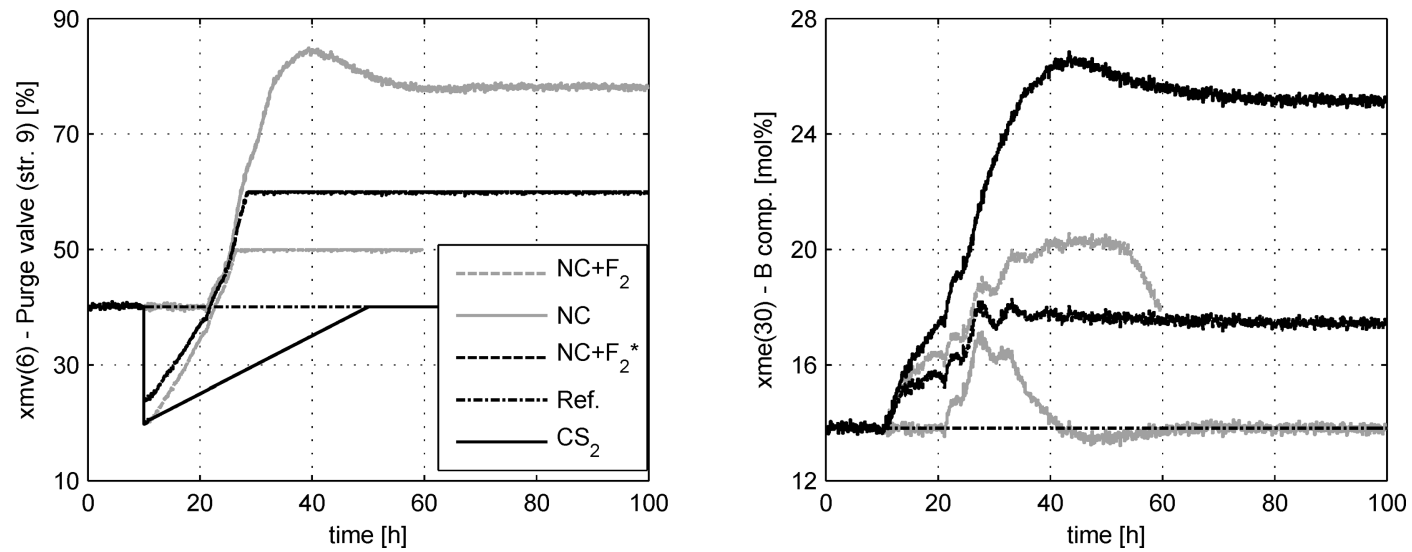

Figure 9. Simulation $S_{2}, \mathrm{xmv}(6)$ and $\mathrm{xme}(30)$ dynamic responses.
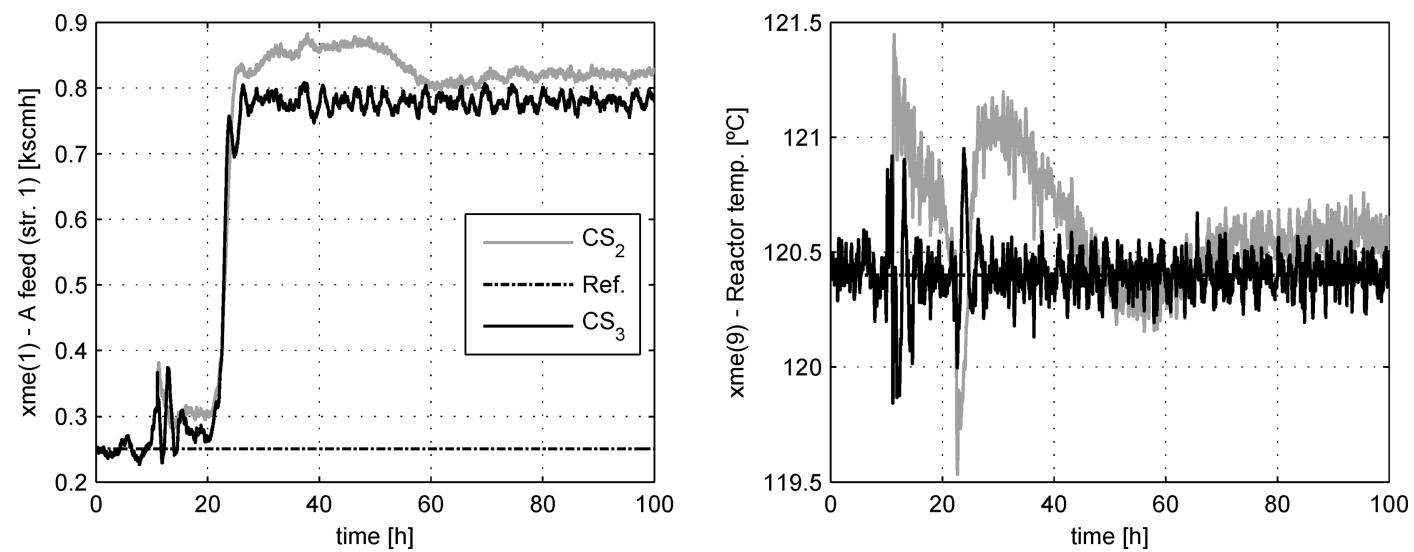

Figure 10. Simulation $S_{3}$, xme(1) and xme(9) dynamic responses.
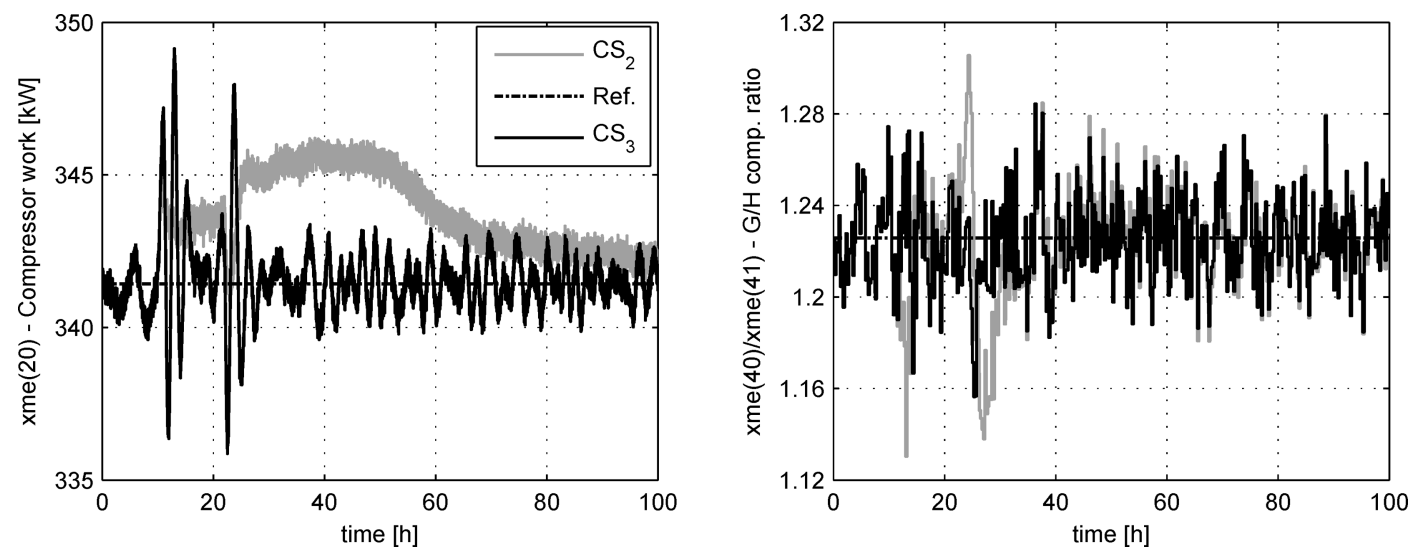

Figure 11. Simulation $S_{3}$, xme $(20)$ and xme $(40) / x m e(41)$ dynamic responses.

$\operatorname{xmv}(4)$ without significant transients during the reconfiguration. In addition, the control energy expenditure is reduced with respect to NC by $16.84 \%$. On the other hand, $\operatorname{EIP}(x m e(30))=$ $-192.65 \%$ since this output is no longer controlled with $\mathrm{CS}_{1}$. However, after the $\mathrm{NC}$ reconfiguration, the system starts controlling $x m e(9)$ and $x m e(20)$. For this reason, a reduction in the corresponding IAE values was obtained: $\operatorname{EIP}(x m e(9))=59.46 \%$ and $\operatorname{EIP}(x m e(20))=71.73 \%$.

The following simulation case $S_{2}$ is disclosed in Figures 8 and 9. As can be seen in Table 6, the structure $\mathrm{CS}_{2}$ presents poor performance with regard to NC. While the EIP index is negative for most outputs, $\mathrm{CS}_{2}$ is able to prevent the shutdown of the process. Figure 9 displays the temporal evolution of the $\mathrm{xmv}(6)-$ xme(30) control loop, which goes out of service when NC is reconfigured, that is, when $\mathrm{CS}_{2}$ is implemented. Figure 9 shows different cases:

- Without considering failures, the $\mathrm{NC}$ rejects $\operatorname{idv}(1)$ and $\operatorname{idv}(2)$ with a good performance. In this case, $\operatorname{xmv}(6)$ presents acceptable evolution without saturations, and xme(30) has zero offset.

- Another scenario considers an effectiveness of $60 \%$ of actuator $\operatorname{xmv}(6)$ (called $\left.F_{2}^{*}\right)$. Here, the saturation of $\operatorname{xmv}(6)$ 

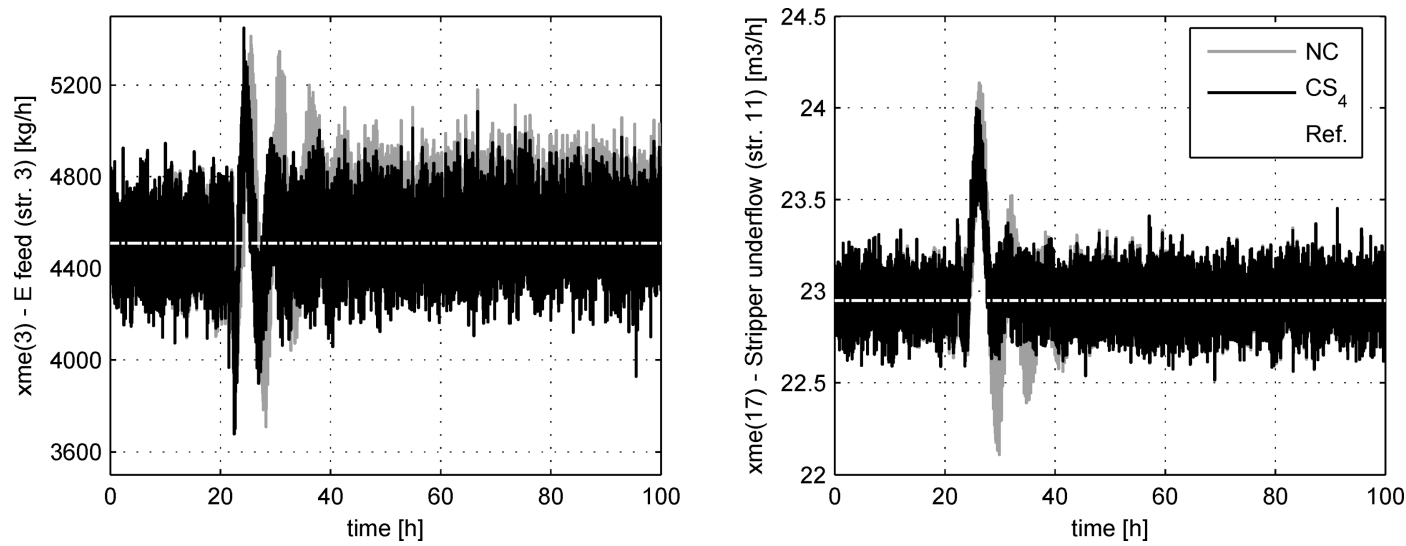

Figure 12. Simulation $S_{4}$, xme(3) and xme(17) dynamic responses.
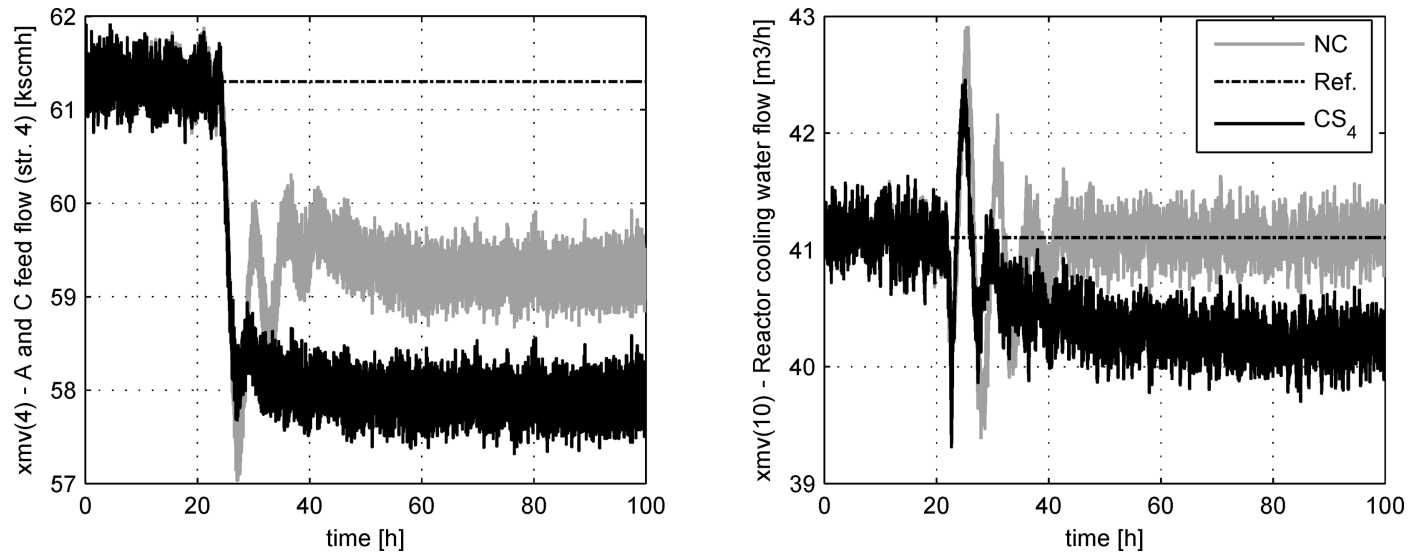

Figure 13. Simulation $S_{4}, \operatorname{xmv}(4)$ and $\operatorname{xmv}(10)$ dynamic responses.
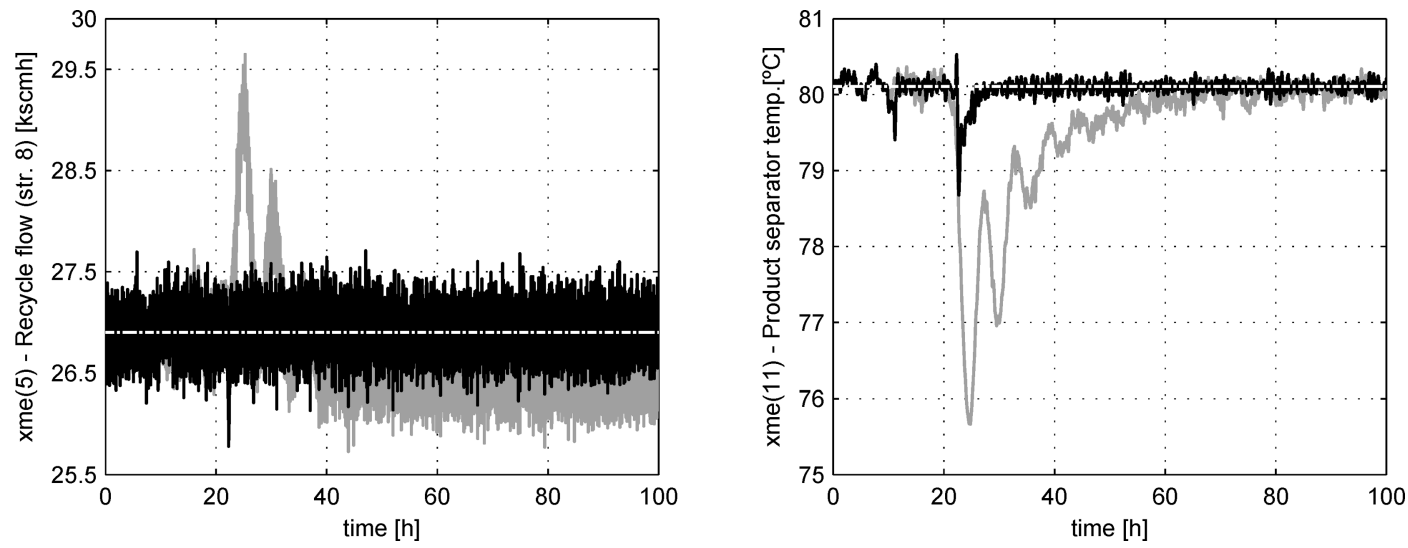

Figure 14. Simulation $S_{5}$, xme(5) and xme(11) dynamic responses.

can be observed due to its effectiveness reduction. While the disturbance is rejected with poor performance, the shutdown of the process does not occur.

- The effect of $F_{2}$ is similar to $F_{2}^{*}$, but a shutdown of the process is produced after the occurrence of $F_{2}(t=10 \mathrm{~h})$, $\operatorname{idv}(2)(t=21 \mathrm{~h})$, and $\operatorname{idv}(1)(t=22 \mathrm{~h})$, given the high reactor pressure (see Figure 8 b).

- When NC is reconfigured, $\operatorname{xmv}(6)$ is no longer controlled with $\mathrm{CS}_{2}$. Thus, this manipulated variable is smoothly set to its nominal operating value in manual mode of the controller. Note the degraded performance of xme(30), which is consistent with its negative EIP value, see Table 6. However, the shutdown of the process is prevented. The shutdown situation is also illustrated in Figure 8, where the temporal evolution of the $\mathrm{xmv}(3)-\mathrm{xme}(7)$ control loop is displayed.

Simulation $S_{3}$ shows that the performance of the system is significantly improved by implementing the alternative structure $\mathrm{CS}_{3}$, which involves five control loops. As indicated in Table 6, most outputs reduce the IAE index with respect to $\mathrm{CS}_{2}$. Figure 10a shows the A feed (stream 1, xme(1)) response, which is not 

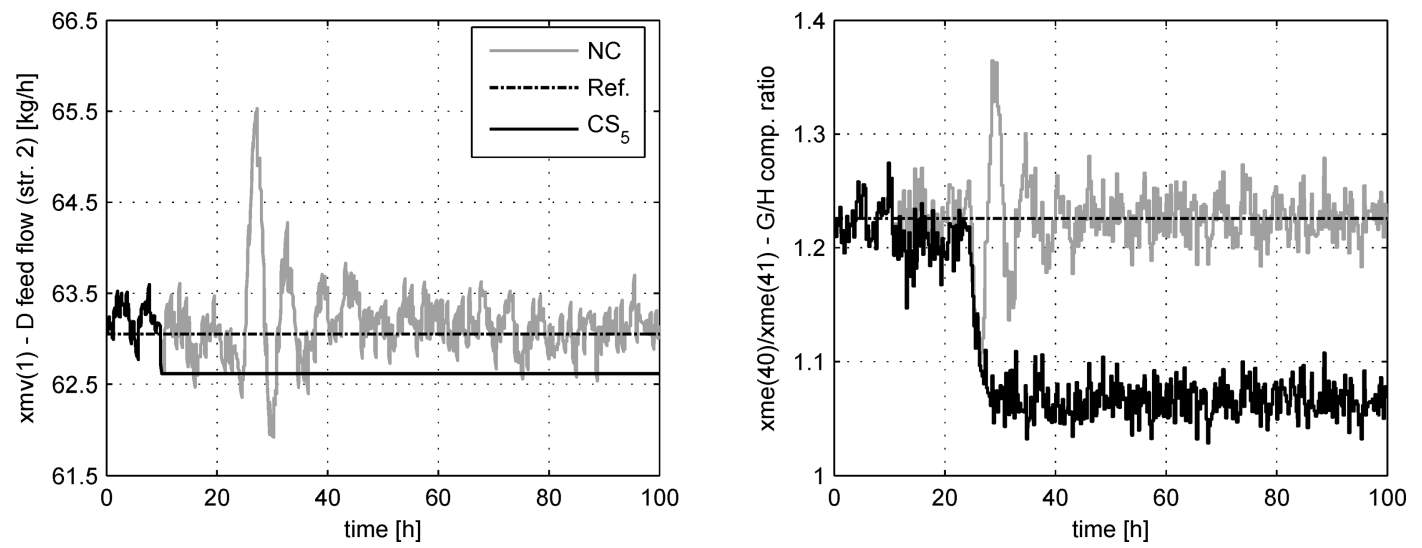

Figure 15. Simulation $S_{5}, \mathrm{xmv}(1)$ and $\mathrm{xme}(40) / \mathrm{xme}(41)$ dynamic responses.

controlled either with $\mathrm{CS}_{2}$ or with $\mathrm{CS}_{3}$. It presents better performance with regard to $\mathrm{CS}_{2}$, with $\operatorname{EIP}(\mathrm{xme}(1))=9.24 \%$. In addition, Figure $10 \mathrm{~b}$ shows the reactor temperature $(\mathrm{xme}(9))$ dynamics. Note that $\operatorname{EIP}(x m e(9))=68.63 \%$ because it is controlled by the $\mathrm{CS}_{3}$ structure. On the other hand, the $\mathrm{CS}_{3}$ solution presents good regulatory behavior subject to $\operatorname{idv}(1)$ and $\operatorname{idv}(2)$, with a reduction in the corresponding IAE value for xme (20) and $\mathrm{xme}_{\mathrm{G} / \mathrm{H}}: \operatorname{EIP}(\mathrm{xme}(20))=62.92 \%$ and $\operatorname{EIP}\left(\mathrm{xme}_{\mathrm{G} / \mathrm{H}}\right)=12.12 \%$, respectively, see Figure 11 .

Another simulation case $S_{4}$ is displayed in Figures 12 and 13 and considers the total failure of actuator $\operatorname{xmv}(6)$. Figure 12 shows the E feed (stream 3, xme(3)) and stripper underflow (stream 11, xme(17)) responses. For these outputs, the IAE index is improved by implementing the $\mathrm{CS}_{4}$ structure: $\operatorname{EIP}(x m e(3))=23.36 \%$ and $\operatorname{EIP}(x m e(17))=13.28 \%$. In general, the overall performance of $\mathrm{CS}_{4}$ is comparable with NC. Note that these structures differ only in one control loop. The obtained EIP values are acceptable, alternating positive and negative values. Furthermore, most manipulated variables show adequate evolution, with less control energy expenditure. Only $x m v(4)$ and $\operatorname{xmv}(10)$ expend more control energy, as shown in Figure 13.

In the last simulation case, $S_{5}$, the dynamic performance of structure $\mathrm{CS}_{5}$ is analyzed. While $\mathrm{CS}_{5}$ is able to manage the total failure of actuators $\operatorname{xmv}(1), \operatorname{xmv}(4)$, and $\operatorname{xmv}(6)$, the system presents poor performance with regard to NC. This is reflected in the EIP index, which is negative for most outputs (see Table 6). In Figure 14, the recycle flow (stream 8, xme(5)) and the product separator temperature $(x m e(11))$ responses are displayed. The $\mathrm{CS}_{5}$ strategy presents good regulatory behavior, with a reduction in the IAE with respect to NC: $\operatorname{EIP}(x m e(5))=56.84 \%$ and $\operatorname{EIP}(x m e(11))=84.24 \%$. This is because these outputs are controlled by the $\mathrm{CS}_{5}$ structure. On the other hand, Figure 15a shows the $\mathrm{D}$ feed flow (stream $2, \operatorname{xmv}(1)$ ) dynamics. Note that a blockade occurs at $t=10 \mathrm{~h}$. After this failure and the reconfiguration of $\mathrm{NC}$ at $t=11 \mathrm{~h}$, the output $\mathrm{xme}_{\mathrm{G} / \mathrm{H}}$ is no longer controlled with $\mathrm{CS}_{5}$. For this reason, an offset is observed in Figure $15 b$, and the corresponding IAE increases: $\operatorname{EIP}\left(\mathrm{xme}_{\mathrm{G} / \mathrm{H}}\right)=$ $-501.20 \%$. The same situation applies for xme(17) and xme(30), see Table 6.

In all cases, it is important to mention that the FTC structure maintains the system stability during the FDD phase, that is, between $t_{F_{i}}$ and $t_{\mathrm{RC}}$ where the process is still controlled by NC. This is due to the procedure utilized for the design of NC, which is based on the fulfillment of a sufficient condition for decentralized integral controllability. ${ }^{9}$

\section{CONCLUSIONS}

From the design and implementation of the proposed reconfigurable FTC, it was possible to increase the system availability by preventing serious situations that would require a process shutdown. It was shown that different types of severe abnormal events were successfully handled by selecting the appropriate hardware redundancies and implementing the corresponding control structures. Their design was addressed offline, thus minimizing the necessary reconfiguration time of the system. The proposed MSV and SSD indexes are exclusively based on the steady-state model of the process and are independent from the control configuration. Different methods and tools were integrated in a multiobjective optimization framework based on genetic algorithm. It consists of a computationally efficient procedure for solving large combinatorial problems like the TE case study, allowing one to systematize the search for candidate solutions.

Despite the contribution, some aspects of the methodology should be improved, namely: (i) the approach is limited to the design of square control structures (i.e., equal number of controlled and manipulated variables), (ii) the proposed formulation does not contemplate the explicit incorporation of constraints, in particular associated with limit values of the manipulated variables, (iii) when the system switches from the nominal controller to an alternative control structure, the difference between the controller outputs could introduce significant transients, and (iv) the approach provides solutions for preconceived failures at the beginning of the design stage. This means that if new failures occur during operation, the system behavior will be unpredictable since it has no ability to perform online design. The above problems motivate the analysis of alternative techniques, such as control allocation, ${ }^{30}$ to improve the approach.

Finally, it is well-known that the equipment design and process configuration constitute causes of control imperfection such as interactions between control loops. ${ }^{31}$ Specifically, an incorrect selection of the process design parameters could greatly affect the process controllability ${ }^{16}$ and consequently the fault-tolerant capability of the system. In this context, future work will be focused on the incorporation of some control aspects into the process design. The objective is to improve the profitability of the process by tolerating different fault cases.

\section{ASSOCIATED CONTENT}

\section{Supporting Information}

The Supporting Information is available free of charge on the ACS Publications website at DOI: 10.1021/acs.iecr.6b01517. 
Implemented SISO PI controllers (PDF)

\section{AUTHOR INFORMATION}

\section{Corresponding Authors}

*E-mail address: luppi@cifasis-conicet.gov.ar (P. A. Luppi). *E-mail: mbasualdo@frro.utn.edu.ar (M. S. Basualdo).

Notes

The authors declare no competing financial interest.

\section{ACKNOWLEDGMENTS}

The authors thank the financial supports from CONICET (Consejo Nacional de Investigaciones Científicas y Técnicas) and UNR-FCEIA (Universidad Nacional de Rosario). The authors also acknowledge the support from UTN-FRRo (Universidad Tecnológica Nacional) and CIFASIS (Centro Internacional Franco Argentino de Ciencias de la Información y de Sistemas).

\section{REFERENCES}

(1) Mhaskar, P.; Liu, J.; Christofides, P. Fault-Tolerant Process Control. Methods and Applications; Springer: London, 2013.

(2) Zhang, Y.; Jiang, J. Bibliographical review on reconfigurable faulttolerant control systems. Annu. Rev. Control 2008, 32, 229-252.

(3) Patton, R. Fault-tolerant Control: The 1997 Situation. 3rd IFAC Symp. Fault Detect., Superv. Saf. Tech. Processes 1997, 1033-1055.

(4) Jiang, J.; Yu, X. Fault-tolerant control systems: A comparative study between active and passive approaches. Annu. Rev. Control 2012, 36, $60-72$.

(5) Jiang, J.; Zhao, Q. Design of Reliable Control Systems Possessing Actuator Redundancies. J. Guid. Control Dynam. 2000, 23, 709-718.

(6) Blanke, M.; Kinnaert, M.; Lunze, J.; Staroswiecki, M. Diagnosis and Fault-Tolerant Control; Springer: Berlin, 2006.

(7) Luppi, P.; Outbib, R; Basualdo, M. Nominal Controller Design Based on Decentralized Integral Controllability in the Framework of Reconfigurable Fault-Tolerant Structures. Ind. Eng. Chem. Res. 2015, 54, $1301-1312$.

(8) Campo, P.; Morari, M. Achievable closed-loop properties of systems under decentralized control: conditions involving the steadystate gain. IEEE Trans. Autom. Control 1994, 39, 932-943.

(9) Skogestad, S.; Morari, M. Variable selection for decentralized control. AIChE Annu. Meet., Washington DC 1988, 128c.

(10) Zumoffen, D. Oversizing analysis in plant-wide control design for industrial processes. Comput. Chem. Eng. 2013, 59, 145-155.

(11) Downs, J.; Skogestad, S. An industrial and academic perspective on plantwide control. Annu. Rev. Control 2011, 35, 99-110.

(12) Luppi, P.; Nieto Degliuomini, L.; Garcia, M.; Basualdo, M. Faulttolerant control design for safe production of hydrogen from bioethanol. Int. J. Hydrogen Energy 2014, 39, 231-248.

(13) Sharifzadeh, M.; Thornhill, N. Optimal selection of control structure using a steady-state inversely controlled process model. Comput. Chem. Eng. 2012, 38, 126-138.

(14) Yu, X.; Jiang, J. Hybrid Fault-Tolerant Flight Control System Design Against Partial Actuator Failures. IEEE T. Contr. Syst. T. 2012, 20, 871-886.

(15) Deb, K. Multi-Objective Optimization Using Evolutionary Algorithms; John Wiley \& Sons: Chichester, U.K., 2001.

(16) Skogestad, S.; Postlethwaite, I. Multivariable Feedback Control. Analysis and Design; John Wiley \& Sons: Chichester, U.K., 2005.

(17) Morari, M. Design of Resilient Processing Plants III. Chem. Eng. Sci. 1983, 38, 1881-1891.

(18) Luyben, W. Process Modeling, Simulation and Control for Chemical Engineers; McGraw-Hill: New York, 1990.

(19) Luppi, P.; Zumoffen, D.; Basualdo, M. Decentralized plantwide control strategy for large-scale processes. Case study: Pulp mill benchmark problem. Comput. Chem. Eng. 2013, 52, 272-285.
(20) Molina, G.; Zumoffen, D.; Basualdo, M. Plant-wide Control Strategy Applied to the Tennessee Eastman Process at Two Operating Points. Comput. Chem. Eng. 2011, 35, 2081-2097.

(21) Nieto Degliuomini, L.; Zumoffen, D.; Basualdo, M. Low cost monitoring system for safe production of hydrogen from bio-ethanol. Int. J. Hydrogen Energy 2013, 38, 13872-13883.

(22) Kariwala, V.; Cao, Y. Branch and Bound method for multiobjective pairing selection. Automatica 2010, 46, 932-936.

(23) Downs, J. J.; Vogel, E. F. A plant-wide industrial process control problem. Comput. Chem. Eng. 1993, 17, 245-255.

(24) Isermann, R. Fault-Diagnosis Applications. Model-Based Condition Monitoring: Actuators, Drives, Machinery, Plants, Sensors, and Faulttolerant Systems; Springer: Berlin, 2011.

(25) Venkatasubramanian, V.; Rengaswamy, R.; Kavuri, S.; Yin, K. A review of process fault detection and diagnosis: Part III: Process history based methods. Comput. Chem. Eng. 2003, 27, 327-346.

(26) Yu, C.; Luyben, W. Design of Multiloop SISO Controllers in Multivariable Processes. Ind. Eng. Chem. Process Des. Dev. 1986, 25, 498-503.

(27) Sakizlis, V.; Perkins, J.; Pistikopoulos, E. Recent advances in optimization-based simultaneous process and control design. Comput. Chem. Eng. 2004, 28, 2069-2086.

(28) Matlab, Global Optimization Toolbox. Multiobjective Optimization; The MathWorks, Inc.: Natick, MA, 2015.

(29) Branke, J.; Deb, K.; Miettinen, K.; Slowinski, R. Multiobjective Optimization. Interactive and Evolutionary Approaches; Springer: Berlin, 2008.

(30) Johansen, T.; Fossen, T. Control allocation. A survey. Automatica 2013, 49, 1087-1103.

(31) Sharifzadeh, M. Integration of process design and control: a review. Chem. Eng. Res. Des. 2013, 91, 2515-2549. 